\title{
TEN NEW AND UPDATED MULTIPLANET SYSTEMS AND A SURVEY OF EXOPLANETARY SYSTEMS
}

\author{
J. T. Wright ${ }^{1}$, S. Upadhyay ${ }^{2}$, G. W. Marcy ${ }^{2}$, D. A. Fischer ${ }^{3}$, Eric B. Ford ${ }^{4}$, and John Asher Johnson ${ }^{5,6}$ \\ ${ }^{1}$ Department of Astronomy, 226 Space Sciences Building, Cornell University, Ithaca, NY 14853, USA; jtwright@astro.cornell.edu \\ ${ }^{2}$ Department of Astronomy, 601 Campbell Hall, University of California, Berkeley, CA 94720-3411, USA \\ ${ }_{3}^{3}$ Department of Physics and Astronomy, San Francisco State University, San Francisco, CA 94132, USA \\ ${ }^{4}$ Department of Astronomy, University of Florida, 211 Bryant Space Science Center, P.O. Box 112055, Gainesville, FL 32611-2055, USA \\ ${ }^{5}$ Institute for Astronomy, University of Hawaii, Honolulu, HI 96822, USA \\ Received 2008 August 16; accepted 2008 October 30; published 2009 March 5
}

\begin{abstract}
We present the latest velocities for ten multiplanet systems, including a re-analysis of archival Keck and Lick data, resulting in improved velocities that supersede our previously published measurements. We derive updated orbital fits for 10 Lick and Keck systems, including two systems (HD 11964, HD 183263) for which we provide confirmation of second planets only tentatively identified elsewhere, and two others (HD 187123 and HD 217107) for which we provide a major revision of the outer planet's orbit. We compile orbital elements from the literature to generate a catalog of the 28 published multiple-planet systems around stars within $200 \mathrm{pc}$. From this catalog we find several intriguing patterns emerging: (1) including those systems with long-term radial velocity trends, at least $28 \%$ of known planetary systems appear to contain multiple planets; (2) planets in multipleplanet systems have somewhat smaller eccentricities than single planets; and (3) the distribution of orbital distances of planets in multiplanet systems and single planets are inconsistent: single-planet systems show a pileup at $P \sim 3$ days and a jump near $1 \mathrm{AU}$, while multiplanet systems show a more uniform distribution in log-period. In addition, among all planetary systems we find the following. (1) There may be an emerging, positive correlation between stellar mass and giant-planet semimajor axis. (2) Exoplanets with $M \sin i>1 M_{\text {Jup }}$ more massive than Jupiter have eccentricities broadly distributed across $0<e<0.5$, while lower mass exoplanets exhibit a distribution peaked near $e=0$.
\end{abstract}

Key words: planetary systems

Online-only material: machine-readable table

\section{INTRODUCTION}

\subsection{The Detection of Multiple-Planet Systems}

The first exoplanetary system known to comprise multiple planets $^{7}$ was the triple system $v$ And (Butler et al. 1999), detected by the radial velocity method just four years after the first confirmed exoplanet, 51 Peg $b$ (Mayor \& Queloz 1995). The subsequent discovery of a second planet orbiting 47 UMa (Fischer et al. 2002a) and the resonant pair of planets orbiting GJ 876 (Marcy et al. 2001) foreshadowed the discovery of more than two dozen systems. Today, $14 \%$ of known host stars of exoplanets within $200 \mathrm{pc}$ are known to be multiple-planet systems, and another $14 \%$ show significant evidence of multiplicity in the form of long-term radial velocity trends.

The recent proliferation of multiple-planet systems is due to the increase in both the velocity precision and duration of the major planet search programs. The increased time baseline has led to the detection of long-period outer companions, the first being the $P=1270 \mathrm{~d}$ planet in the multiple system $v$ And (Butler et al. 1999). Today, the 10+ year baseline of high-precision (< $5 \mathrm{~m} \mathrm{~s}^{-1}$ ) radial velocity planet searches means that most planets with $a<3$ AU now have multiple complete orbits observed, improving their detectability. Even planets with $a>4 \mathrm{AU}$ which have not yet completed a single orbit can sometimes have well-constrained minimum masses $(M \sin i)$, as in the case of HD $187123 c$ (Wright et al. 2007).

\footnotetext{
6 NSF postdoctoral fellow.

7 Prior to this, Wolszczan \& Frail (1992) detected three extraordinary planets orbiting the pulsar PSR $1257+12$. Here, we restrict the discussion to systems orbiting nearby, ordinary stars.
}

Radial velocity precision has steadily improved toward and below $1 \mathrm{~m} \mathrm{~s}^{-1}$ with High Resolution Echelle Spectrometer (HIRES) at the Keck Observatory and with the HARPS spectrograph at La Silla (Pepe et al. 2003; Wright et al. 2007). This has allowed for the detection of ever weaker signals and led to the discovery of some of the lowest-mass planets known, including (among others): 55 Cnc $e$ (McArthur et al. 2004), GJ $876 d$ (Rivera et al. 2005), the triple system HD 69830 (Lovis et al. 2006), and $\mu$ Ara $d$ (Santos et al. 2004). We list all published multiple-planet systems within $200 \mathrm{pc}$ in Table 1, a list which benefits from updated and improved velocities of 10 of the systems provided in Table 2. We present a graphical overview of these multiplanet systems in Figure 1.

\subsection{Multiple-Planet Systems and Planet Formation Theory}

Multiple-planet systems are of special interest to test theoretical models of planet formation, dynamics, and final architectures. To date, over 230 planets have been discovered orbiting 205 main sequence stars within 200 pc, $14 \%$ of which harbor multiple planets with well-constrained minimum masses and periods. With 67 such planets members of multiplanet systems, we may now make statistically significant comparisons between properties of planets in single-planet systems with those in multiplanet systems.

The known planet population is remarkably diverse with properties that bear on planet formation theory. Theories to explain the semimajor axis distributions and eccentricity distributions of planets have especially benefitted from the constraints that multiplanet systems impose. Most planet formation theories are based on the core accretion model that begins with a disk of dust and gas where the dust particles collide and grow to form rock- 
Table 1

List of Exoplanets in Multiplanet Systems

\begin{tabular}{|c|c|c|c|c|c|c|c|c|c|c|c|c|c|}
\hline Name & & $\begin{array}{l}\text { Per. } \\
\text { (d) }\end{array}$ & $\begin{array}{c}K \\
\left(\mathrm{~m} \mathrm{~s}^{-1}\right) \\
\end{array}$ & $e^{\mathrm{a}}$ & $\begin{array}{l}\omega^{\mathrm{a}} \\
\left({ }^{\circ}\right) \\
\end{array}$ & $\begin{array}{c}T_{p} \\
(\mathrm{JD}-2440000) \\
\end{array}$ & $\begin{array}{l}M \sin i \\
\left(M_{\text {Jup }}\right)\end{array}$ & $\begin{array}{c}a \\
(\mathrm{AU})\end{array}$ & $\begin{array}{c}\mathrm{rms} \\
\left(\mathrm{m} \mathrm{s}^{-1}\right)\end{array}$ & $\sqrt{\chi_{v}^{2}}$ & $N_{\text {obs }}$ & Note & Reference $^{b}$ \\
\hline \multirow[t]{3}{*}{$v$ And } & $b$ & $4.617136(47)$ & $68.2(1.1)$ & $0.013(16)$ & 51 & $14425.02(64)$ & $0.672(56)$ & $0.0595(34)$ & 14 & 1.6 & 284 & & \\
\hline & $c$ & $241.33(20)$ & $53.6(1.4)$ & $0.224(21)$ & $250.8(4.9)$ & $14265.57(64)$ & $1.92(16)$ & $0.832(48)$ & 14 & 1.6 & 284 & & \\
\hline & $d$ & $-1278.1(2.9)$ & $66.7(1.4)$ & $0.267(21)$ & $269.7(6.4)$ & $13937.73(64)$ & $4.13(35)$ & $2.53(15)$ & 14 & 1.6 & 284 & & \\
\hline \multirow[t]{2}{*}{ HD 11964} & $b$ & 1945(26) & $9.41(39)$ & $0.041(47)$ & 155 & $14170(380)$ & $0.622(56)$ & $3.16(19)$ & 3.1 & 1.1 & 119 & & \\
\hline & $c$ & $37.910(41)$ & $4.65(59)$ & $0.30(17)$ & 102 & $14370(380)$ & $0.0788(97)$ & $0.229(13)$ & 3.1 & 1.1 & 119 & & \\
\hline \multirow[t]{2}{*}{ HD 12661} & $b$ & $262.709(83)$ & $73.56(56)$ & $0.3768(77)$ & $296.0(1.5)$ & $14152.76(87)$ & $2.30(19)$ & $0.831(48)$ & 5.1 & 1.0 & 107 & & \\
\hline & $c$ & $1708(14)$ & $30.41(62)$ & $0.031(22)$ & 165 & $16153.42(87)$ & $1.92(16)$ & $2.90(17)$ & 5.1 & 1.0 & 107 & & \\
\hline \multirow[t]{2}{*}{ HIP 14810} & $b$ & $6.6742(20)$ & $428.3(3.0)$ & $0.1470(60)$ & $158.6(2.0)$ & $13694.588(40)$ & $3.91(32)$ & $0.0692(40)$ & 5.1 & 1.4 & 30 & & $\mathrm{Wr} 7$ \\
\hline & $c$ & $95.2847(20)$ & $37.4(3.0)$ & $0.4091(60)$ & $354.2(2.0)$ & $13679.585(40)$ & $0.762(83)$ & $0.407(23)$ & 5.1 & 1.4 & 30 & & Wr7 \\
\hline \multirow[t]{3}{*}{ HD 37124} & $b$ & 154.46 & 27.5 & 0.055 & 140.5 & 10000.11 & $0.64(11)$ & $0.529(31)$ & 18 & 1.9 & 52 & & Vo5 \\
\hline & $c$ & 2295.00 & 12.2 & 0.2 & 266.0 & 9606.00 & $0.683(88)$ & $3.19(18)$ & 18 & 1.9 & 52 & & Vo5 \\
\hline & $d$ & 843.60 & 15.4 & 0.140 & 314.3 & 9409.40 & $0.624(63)$ & $1.639(95)$ & 5.1 & 1.1 & 52 & & Vo5 \\
\hline \multirow[t]{2}{*}{ HD 38529} & $b$ & $14.31020(81)$ & $57.0(1.2)$ & $0.244(28)$ & $95.4(5.8)$ & $14384.8(8.7)$ & $0.856(72)$ & $0.1313(76)$ & 12 & 1.8 & 175 & & \\
\hline & $c$ & $2146.1(5.5)$ & $169.0(1.5)$ & $0.3551(74)$ & $17.9(1.6)$ & $12255.9(8.4)$ & $13.1(1.1)$ & $3.72(21)$ & 12 & 1.8 & 175 & & \\
\hline \multirow[t]{3}{*}{ HD 40307} & $b$ & $4.31150(60)$ & $1.97(11)$ & 0 & 0 & $14562.770(80)$ & 0.0133 & $0.0475(27)$ & 0.85 & 1.6 & 135 & $\mathrm{c}$ & My8 \\
\hline & $c$ & $9.6200(20)$ & $2.47(11)$ & 0 & 0 & $14551.53(15)$ & 0.0217 & $0.0811(47)$ & 0.85 & 1.6 & 135 & $\mathrm{c}$ & My8 \\
\hline & $d$ & $20.460(10)$ & $4.55(12)$ & 0 & 0 & $14532.42(29)$ & 0.0514 & $0.1342(77)$ & 0.85 & 1.6 & 135 & $\mathrm{c}$ & My8 \\
\hline \multirow[t]{2}{*}{ HD 60532} & $b$ & $201.30(60)$ & $29.3(1.4)$ & $0.280(30)$ & $-8.1(4.9)$ & $13987.0(2.0)$ & $1.03(16)$ & $0.759(44)$ & 4.4 & 2.1 & 147 & & Ds8 \\
\hline & $c$ & $604.0(9.0)$ & $46.4(1.7)$ & $0.020(20)$ & $-209(92)$ & $13730(160)$ & $2.46(36)$ & $1.580(93)$ & 4.4 & 2.1 & 147 & & Ds8 \\
\hline \multirow[t]{3}{*}{ HD 69830} & $b$ & $8.6670(30)$ & $3.51(15)$ & $0.100(40)$ & $340(26)$ & $13496.80(60)$ & $0.0322(45)$ & $0.0789(46)$ & 0.81 & 1.1 & 74 & & Lv6 \\
\hline & $c$ & $31.560(40)$ & $2.66(16)$ & $0.130(60)$ & $221(35)$ & $13469.6(2.8)$ & $0.0374(52)$ & $0.187(11)$ & 0.81 & 1.1 & 74 & & Lv6 \\
\hline & $d$ & $197.0(3.0)$ & $2.20(19)$ & $0.070(70)$ & $224(61)$ & $13358(34)$ & $0.0573(80)$ & $0.633(37)$ & 0.81 & 1.1 & 74 & & Lv6 \\
\hline HD 73526 & $b$ & $188.3(0.9)$ & $\ldots$ & $0.19(05)$ & $\ldots$ & .. & $2.07(16)$ & $0.66(5)$ & 7.9 & 1.3 & 30 & d & $\mathrm{T} 6$ \\
\hline & $c$ & $377.8(2.4)$ & $\ldots$ & $0.14(9)$ & & & $2.5(3)$ & $1.05(8)$ & 7.9 & 1.3 & 30 & d & T6 \\
\hline HD 74156 & $b$ & $51.643(11)$ & $112.0(1.9)$ & $0.6360(91)$ & $181.5(1.4)$ & $11981.321(91)$ & $1.80(26)$ & $0.290(17)$ & 11 & 1.3 & 95 & $\mathrm{e}$ & $\mathrm{Nf} 4$ \\
\hline & $c$ & $2025(11)$ & $104.0(5.5)$ & $0.583(39)$ & $242.4(4.0)$ & 10901(10) & $6.00(95)$ & $3.35(19)$ & 11 & 1.3 & 95 & e & $\mathrm{Nf} 4$ \\
\hline & $d$ & $276.4(3.6)$ & $10.5(1.2)$ & $0.25(11)$ & $167(27)$ & $678(44)$ & $0.371(80)$ & $0.886(52)$ & 6.0 & 0.94 & 242 & $\mathrm{e}$ & $\mathrm{Be} 8$ \\
\hline $55 \mathrm{Cnc}$ & $b$ & $14.65126(70)$ & $71.84(41)$ & $0.0159(80)$ & $164(30)$ & $7572.0(1.2)$ & $0.82(12)$ & $0.1138(66)$ & 7.71 & 2.012 & 636 & f & Fi8 \\
\hline & $c$ & $44.3787(70)$ & $10.06(43)$ & $0.053(52)$ & $57(29)$ & $7547.5(3.3)$ & $0.165(26)$ & $0.238(14)$ & 7.71 & 2.012 & 636 & $\mathrm{f}$ & Fi8 \\
\hline & $d$ & $5370(230)$ & $47.2(1.8)$ & $0.063(30)$ & $163(32)$ & $6860(230)$ & $3.84(58)$ & $5.84(39)$ & 7.71 & 2.012 & 636 & f & Fi8 \\
\hline & $e$ & $2.79674(10)$ & $3.73(53)$ & $0.264(60)$ & $157(38)$ & $7578.21590(10)$ & $0.0235(49)$ & $0.0377(22)$ & 7.71 & 2.012 & 636 & f & Fi8 \\
\hline & $f$ & $260.7(1.1)$ & $4.75(60)$ & $0.00(20)$ & $206(60)$ & $7488.0(1.1)$ & $0.141(39)$ & $0.775(45)$ & 7.71 & 2.012 & 636 & f & Fi8 \\
\hline HD 82943 & $b$ & 219.3 & 66.0 & 0.359 & 127 & $\ldots$ & 2.01 & 0.752 & 7.9 & 1.4 & 155 & g & Le6 \\
\hline & $c$ & 441.2 & 43.6 & 0.219 & 284 & & 1.75 & 1.20 & 7.9 & 1.4 & 155 & $\mathrm{~g}$ & Le6 \\
\hline $47 \mathrm{UMa}$ & $b$ & $1089.0(2.9)$ & $49.3(1.2)$ & $0.061(14)$ & 102 & $10356(34)$ & $2.63(22)$ & $2.13(12)$ & 7.4 & 1.0 & 90 & & Fi2 \\
\hline & $c$ & $2594(90)$ & 11.1(1.1) & $0.00(12)$ & $127(56)$ & $11360(500)$ & $0.792(92)$ & $3.79(24)$ & 7.4 & 1.0 & 90 & & Fi2 \\
\hline HD 102272 & $b$ & $127.58(30)$ & $155.5(5.6)$ & $0.050(40)$ & $118(58)$ & $12146(64)$ & $5.91(89)$ & $0.615(36)$ & 15 & 0.87 & 37 & & $\mathrm{Ni} 8$ \\
\hline & $c$ & $520(26)$ & $59(11)$ & $0.680(60)$ & $320(10)$ & $14140(260)$ & $2.63(66)$ & $1.57(11)$ & 15 & 0.87 & 37 & & $\mathrm{Ni} 8$ \\
\hline HD 108874 & $b$ & $394.48(60)$ & $37.3(1.1)$ & $0.128(22)$ & $219.4(9.3)$ & $14045(49)$ & $1.34(11)$ & $1.053(61)$ & 4.0 & 1.0 & 55 & & \\
\hline & $c$ & $1680(24)$ & $18.90(72)$ & $0.273(40)$ & $10(11)$ & 12797(49) & $1.064(99)$ & $2.77(16)$ & 4.0 & 1.0 & 55 & & \\
\hline HD 128311 & $b$ & $458.6(6.8)$ & $66.8(8.7)$ & $0.25(10)$ & $111(36)$ & $10210.9(7.6)$ & $2.19(27)$ & $1.100(65)$ & 18 & 1.9 & & & Vo5 \\
\hline & $c$ & $928(18)$ & $76.2(4.6)$ & $0.170(90)$ & $200(150)$ & $10010(400)$ & $3.22(29)$ & $1.76(11)$ & 18 & 1.9 & & & Vo5 \\
\hline GJ 581 & $b$ & $5.36870(30)$ & $12.42(19)$ & 0 & 0 & $12999.990(50)$ & 0.0490 & $0.0406(23)$ & 1.3 & 1.8 & 50 & & U7 \\
\hline & $c$ & $12.9310(70)$ & $3.01(16)$ & 0 & 0 & $12996.74(45)$ & 0.0159 & $0.0730(42)$ & 1.3 & 1.8 & 50 & & U7 \\
\hline & $d$ & $83.40(40)$ & $2.67(16)$ & 0 & 0 & $12954.1(3.7)$ & 0.0263 & $0.253(15)$ & 1.3 & 1.8 & 50 & & U7 \\
\hline HD 155358 & $b$ & $195.0(1.1)$ & $34.6(3.0)$ & $0.112(37)$ & $162(20)$ & $13950(10)$ & $0.89(15)$ & $0.628(36)$ & 6.0 & 1.1 & 71 & & $\mathrm{Cc} 7$ \\
\hline & $c$ & $530(27)$ & 14.1(1.6) & $0.18(17)$ & $279(38)$ & $14420(79)$ & $0.50(13)$ & $1.224(87)$ & 6.0 & 1.1 & 71 & & $\mathrm{Cc} 7$ \\
\hline$\mu$ Ara & $b$ & $630.0(6.2)$ & $37.4(1.6)$ & $0.271(40)$ & $259.8(7.4)$ & $10881(28)$ & $1.67(17)$ & $1.510(88)$ & 4.7 & 1.1 & 108 & & Bu6 \\
\hline & $c$ & $2490(100)$ & $18.1(1.1)$ & $0.463(53)$ & $183.8(7.9)$ & $11030(110)$ & $1.18(12)$ & $3.78(25)$ & 4.7 & 1.1 & 108 & & Bu6 \\
\hline & $d$ & $9.6386(15)$ & $3.06(13)$ & $0.172(40)$ & $213(13)$ & $12991.10(40)$ & $0.0347(53)$ & $0.0930(54)$ & 1.7 & 1.1 & 171 & & Pp7 \\
\hline & $e$ & $310.55(83)$ & $14.91(59)$ & $0.067(12)$ & $189.6(9.4)$ & $12708.7(8.3)$ & $0.546(80)$ & $0.942(54)$ & 1.7 & 1.1 & 171 & & Pp7 \\
\hline HD 168443 & $b$ & $58.11212(48)$ & $475.54(88)$ & $0.5295(11)$ & $172.95(13)$ & $14347.728(20)$ & $8.01(65)$ & $0.300(17)$ & 3.5 & 0.84 & 112 & h & \\
\hline & $c$ & $1748.2(1.0)$ & $298.14(61)$ & $0.2122(20)$ & $64.68(52)$ & $13769.768(21)$ & $18.1(1.5)$ & $2.91(17)$ & 3.5 & 0.84 & 112 & h & \\
\hline HD 169830 & $b$ & $225.62(22)$ & $80.70(90)$ & $0.310(10)$ & $148.0(2.0)$ & $11923.0(1.0)$ & $2.92(25)$ & $0.817(47)$ & 8.9 & $\ldots$ & 112 & & My4 \\
\hline & $c$ & $2100(260)$ & $54.3(3.6)$ & $0.330(20)$ & $252.0(8.0)$ & $12516(25)$ & $4.10(41)$ & $3.62(42)$ & 8.9 & $\ldots$ & 112 & & My4 \\
\hline HD 183263 & $b$ & $626.5(1.1)$ & $84.0(3.7)$ & $0.3567(90)$ & $233.5(2.3)$ & $12111.7(3.0)$ & $3.67(30)$ & $1.510(87)$ & 3.7 & 1.2 & 41 & & \\
\hline & $c$ & $3070(110)$ & $46.3(3.7)$ & $0.239(64)$ & $345(12)$ & 11971(94) & $3.57(55)$ & $4.35(28)$ & 3.7 & 1.2 & 41 & & \\
\hline HD 187123 & $b$ & $3.0965828(78)$ & $69.40(45)$ & $0.0103(59)$ & 25 & $14343.12(31)$ & $0.523(43)$ & $0.0426(25)$ & 2.5 & 0.65 & 76 & & \\
\hline & $c$ & $3810(420)$ & $25.5(1.5)$ & $0.252(33)$ & $243(19)$ & $13580.04(30)$ & $1.99(25)$ & $4.89(53)$ & 2.5 & 0.65 & 76 & & \\
\hline HD 190360 & $b$ & $2915(29)$ & $23.24(46)$ & $0.313(19)$ & $12.9(4.0)$ & $13542(31)$ & $1.56(13)$ & $4.01(23)$ & 3.1 & 0.84 & 107 & & \\
\hline & $c$ & $17.1110(48)$ & $4.84(51)$ & $0.237(82)$ & $5(26)$ & $14390(31)$ & $0.0600(76)$ & $0.1304(75)$ & 3.1 & 0.84 & 107 & & \\
\hline HD 202206 & $b$ & $255.870(60)$ & $564.8(1.3)$ & $0.4350(10)$ & $161.18(30)$ & $\ldots$ & $17.3(2.4)$ & $0.823(48)$ & 9.6 & 1.5 & & $\mathrm{i}$ & $\mathrm{Cr} 5$ \\
\hline & $c$ & $1383(18)$ & $42.0(1.5)$ & $0.267(21)$ & $79.0(6.7)$ & & $2.40(35)$ & $2.52(15)$ & 9.6 & 1.5 & & $\mathrm{i}$ & $\mathrm{Cr} 5$ \\
\hline GJ 876 & $b$ & $60.940(13)$ & $212.60(76)$ & $0.0249(26)$ & $175.7(6.0)$ & & $1.93(27)$ & $0.208(12)$ & 4.6 & 1.2 & 155 & $\mathrm{j}$ & R5 \\
\hline & $c$ & $30.340(13)$ & $88.36(72)$ & $0.2243(13)$ & $198.30(90)$ & $\ldots$ & $0.619(88)$ & $0.1303(75)$ & 4.6 & 1.2 & 155 & $\mathrm{j}$ & $\mathrm{R} 5$ \\
\hline & $d$ & $1.937760(70)$ & $6.46(59)$ & 0 & $\ldots$ & $\ldots$ & $0.0185(31)$ & $0.0208(12)$ & 4.6 & 1.2 & 155 & $\mathrm{j}$ & R5 \\
\hline
\end{tabular}


Table 1

(Continued)

\begin{tabular}{|c|c|c|c|c|c|c|c|c|c|c|c|c|c|}
\hline Name & & $\begin{array}{l}\text { Per. } \\
\text { (d) }\end{array}$ & $\begin{array}{c}K \\
\left(\mathrm{~m} \mathrm{~s}^{-1}\right)\end{array}$ & $e^{\mathrm{a}}$ & $\begin{array}{l}\omega^{\mathrm{a}} \\
\left({ }^{\circ}\right)\end{array}$ & $\begin{array}{c}T_{p} \\
(\mathrm{JD}-2440000)\end{array}$ & $\begin{array}{l}M \sin i \\
\left(M_{\mathrm{Jup}}\right)\end{array}$ & $\begin{array}{c}a \\
(\mathrm{AU})\end{array}$ & $\begin{array}{c}\mathrm{rms} \\
\left(\mathrm{m} \mathrm{s}^{-1}\right)\end{array}$ & $\sqrt{\chi_{v}^{2}}$ & $N_{\text {obs }}$ & Note & Reference $^{b}$ \\
\hline \multirow[t]{2}{*}{ HD 217107} & $b$ & $7.126816(39)$ & $139.20(92)$ & $0.1267(52)$ & $24.4(3.0)$ & $14396(39)$ & $1.39(11)$ & $0.0748(43)$ & 11 & 2.1 & 207 & & \\
\hline & $c$ & $4270(220)$ & $35.7(1.3)$ & $0.517(33)$ & $198.6(6.0)$ & $11106(39)$ & $2.60(15)$ & $5.32(38)$ & 11 & 2.1 & 207 & & \\
\hline
\end{tabular}

Notes. For succinctness, we express uncertainties using parenthetical notation, where the least significant digit of the uncertainty, in parentheses, and that of the quantity are understood to have the same place value. Thus, " $0.100(20)$ " indicates " $0.100 \pm 0.020$," "1.0(2.0)" indicates " $1.0 \pm 2.0$," and " $1(20)$ " indicates " $1 \pm 20$."

${ }^{a}$ When the uncertainty in $e$ is comparable to $e$, uncertainties in $\omega$ and $e$ become non-Gaussian. See Butler et al. (2006) for details.

${ }^{b}$ References indicate which orbital parameters are taken from the literature as follows-Be8: Bean et al. (2008); Bu6: Butler et al. (2006); Cc7: Cochran et al. (2007); Cr5: Correia et al. (2005); Ds8: Desort et al. (2008); Fi2: Fischer et al. (2002b); Fi8: Fischer et al. (2008); Le6: Lee et al. (2006); Lv6: Lovis et al. (2006); My4: Mayor et al. (2004); My8: Mayor et al. (2009); Ni8: Niedzielski et al. (2008); Nf4: Naef et al. (2004); Pp7: Pepe et al. (2007); R5: Rivera et al. (2005); T6: Tinney et al. (2006); U7: Udry et al. (2007); Vo5: Vogt et al.(2005); Wr7: Wright et al. (2007). All other orbital solutions are new Keplerian (kinematic) fits to the data in Table 2. ${ }^{c}$ The fit for this system includes a trend of $-0.51 \pm 0.1 \mathrm{~m} \mathrm{~s}^{-1} \mathrm{yr}^{-1}$.

${ }^{\mathrm{d}}$ The planets in HD 73526 are in a 2:1 mean motion resonance, and planet-planet interactions are important, rendering Keplerian elements inadequate. In addition to the elements reported here, Tinney et al. (2006) report a mean anomaly to be $86^{\circ} \pm 13^{\circ}$ and $82^{\circ} \pm 27^{\circ}$ at a Julian Date of 2451212.1302 . There is considerable degeneracy between $K$ and $e$ because the orbital period of HD $73526 c$ differs from 1 year by only $12 \mathrm{~d}$.

${ }^{\mathrm{e}}$ Barnes et al. (2008) found that the orbit presented in Bean et al. (2008) for the $d$ component is unstable, and provide multiple stable solutions without uncertainties in the orbital parameters.

${ }^{f}$ Planet-planet interactions are strong in 55 Cnc. The osculating orbital elements here are from the dynamical fit at Julian Date 2447578.730 of Fischer et al. (2008). That work puts no errors on these parameters, however, so the errors quoted here are those from the Keplerian (kinematic) fit there.

$\mathrm{g}$ The exoplanets in HD 82946 have significant interactions, which render Keplerian orbital elements inadequate for describing their orbits, since these elements are time variable. Lee et al. (2006) report the mean anomaly of the inner and outer planets to be $353^{\circ}$ and $207^{\circ}$, respectively, at a Julian Date of 2451185.1.

$\mathrm{h}$ This solution includes a linear trend with magnitude $-3.08 \pm 0.16 \mathrm{~m} \mathrm{~s}^{-1} \mathrm{yr}^{-1}$.

i The exoplanets in HD 202206 have significant interactions, which renders Keplerian orbital elements inadequate for describing their orbits, since these elements are time variable. Correia et al. (2005) report the mean longitude to be $266.23^{\circ} \pm 0.06^{\circ}$ and $30.59^{\circ} \pm 2.84^{\circ}$ for the inner and outer planets, respectively, at a Julian Date of 2452250 .

j The outer two exoplanets GJ 876 have significant interactions, which renders Keplerian orbital elements inadequate for describing their orbits, since these elements are time variable. Rivera et al. (2005) report the mean anomaly of the planets to be $M_{d}=309.5^{\circ} \pm 5.1^{\circ}, M_{c}=308.5^{\circ} \pm 1.4^{\circ}$, and $M_{b}=175.5^{\circ} \pm 6.0^{\circ}$, respectively, at a Julian Date of 2452490 . The solution quoted here assumes $i=90^{\circ}$.

Table 2

Updated RV Data for Multiple-Planet Systems

\begin{tabular}{lrccc}
\hline \hline Star & $\begin{array}{c}\text { Time } \\
(\mathrm{JD}-2440000)\end{array}$ & $\begin{array}{c}\text { Radial Velocity } \\
\left(\mathrm{m} \mathrm{s}^{-1}\right)\end{array}$ & $\begin{array}{c}\text { Unc. } \\
\left(\mathrm{m} \mathrm{s}^{-1}\right)\end{array}$ & Telescope \\
\hline upsilon And & 9680.753973 & -124.5 & 6.7 & $\mathrm{~L}$ \\
upsilon And & 9942.007812 & 19.2 & 9.2 & $\mathrm{~L}$ \\
upsilon And & 9969.979618 & -20.2 & 6.5 & $\mathrm{~L}$ \\
upsilon And & 9984.852774 & -53.0 & 6.9 & $\mathrm{~L}$ \\
upsilon And & 10032.741293 & 30.6 & 8.0 & $\mathrm{~L}$ \\
upsilon And & 10056.841914 & 49.7 & 6.4 & $\mathrm{~L}$ \\
upsilon And & 10068.586022 & -58.1 & 7.2 & $\mathrm{~L}$ \\
upsilon And & 10068.773306 & -60.6 & 7.4 & $\mathrm{~L}$ \\
upsilon And & 10069.598633 & 4 & 12 & $\mathrm{~L}$ \\
upsilon And & 10072.602539 & -89 & 10 & $\mathrm{~L}$ \\
\hline
\end{tabular}

(This table is available in its entirety in a machine-readable form in the online journal. A portion is shown here for guidance regarding its form and content.)

ice planetary cores (Aarseth et al. 1993; Kokubo \& Ida 2002; Levison et al. 1998). If a core becomes massive enough while gas remains in the disk, it gravitationally accretes the nearby gas and rapidly increases in mass (Bodenheimer et al. 2003; Pollack et al. 1996). Such gas planets should form preferentially beyond the "ice line" (near 3 AU for solar-type stars), where ices can participate in the initial planetary cores. This appears inconsistent with the observation that about $20 \%$ of known exoplanets orbit within $0.1 \mathrm{AU}$, where there should be too little ice in the protoplanetary disk for massive cores to form quickly.

Thus, it appears that short-period planets form farther out and migrate inwards to their final semimajor axis (Papaloizou 2005; Tanaka \& Ward 2004; Trilling et al. 2002). The discovery of at least five systems in or near mean motion resonance (viz. GJ 876, 55 Cnc, HD 82943, HD 73526, and HD 128311) may lend support to the migration hypothesis (Nelson \& Papaloizou 2002; Lee et al. 2006; Marzari et al. 2005). Such resonances are difficult to explain if planets form in situ, but hydrodynamical simulations and $n$-body simulations with externally applied damping (Kley et al. 2005; Bryden et al. 2000; Chiang \& Murray 2002; D'Angelo et al. 2003; Ida \& Lin 2004) show that resonance capture occurs if planets undergo significant migration at different rates, passing through mean motion resonances. Also, although there are confirmed 2:1 mean motion resonances, other period ratios may occur (Kley et al. 2004; Nelson \& Papaloizou 2002; Laughlin \& Chambers 2002). Future discoveries of multiplanet systems may shed further light on the resonance capture process and on planet formation dynamics.

Multiplanet systems also provide hints about the wide distribution of exoplanet eccentricities and we are beginning to identify different processes that drive some planets to large eccentricities, while damping others to moderate and low eccentricities (Murray 2003; Barnes \& Quinn 2004). Ford (2006) suggests that planet-planet scattering augments eccentricities, and that after the era of strong planet-planet scattering, interactions with the remaining planetesimals will damp these eccentricities to the observed distribution. One may hope to detect some signature of these processes in exoplanet data. Indeed, the configuration of the $v$ Andromedae three-planet system may be explained by a planet-planet scattering event that ejected a fourth planet from the vicinity, as this system carries a signature of sudden perturbation (Ford et al. 2005). HD 128311, a two-planet system, also appears to carry a signature of similar scattering (Sándor \& Kley 2006).

Models of planet formation in protoplanetary disks point to the disk viscosities and lifetimes that are required to produce some observed systems. There is some inconsistency between 


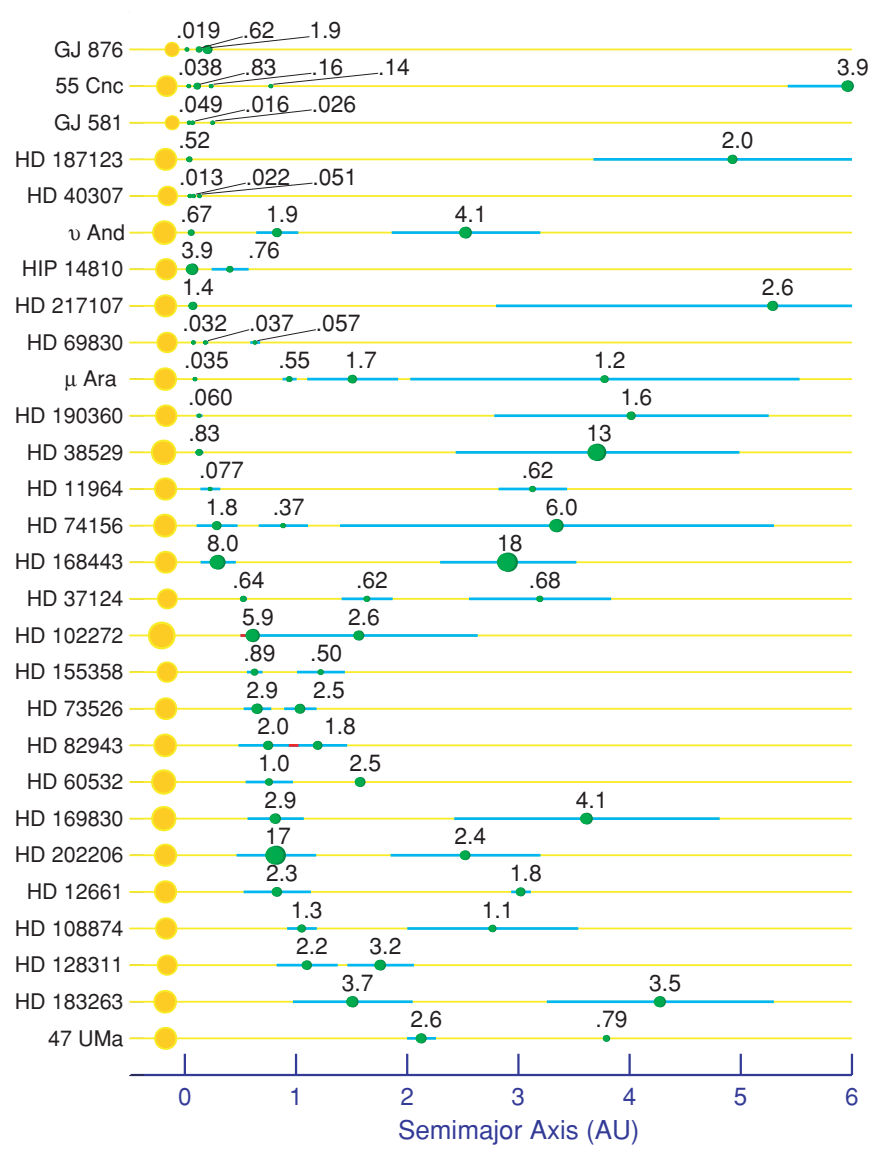

Figure 1. Chart of semimajor axes and minimum masses for the 28 known multiplanet systems. The diameters depicted for planets are proportional the cube root of the planetary $M \sin i$. The periapse to apoapse excursion is shown by a horizontal line intersecting the planet. The diameters depicted for stars are proportional the cube root of the stellar mass.

the required disk behavior and that predicted by hydrodynamical theories (Kley et al. 2004). Simulations that include multiple protoplanets interacting with a protoplanet swarm show that most protoplanets will eventually accrete on to the star, leading to a planet occurrence rate lower than that observed (Cresswell \& Nelson 2006).

The increasing number of characterized multiplanet systems should help constrain these theories. We present the current catalog of multiple-planet systems with the most recent radial velocity data in hopes of offering theorists a thorough overview of current state of observations, and an opportunity to propose new tests of planet formation theories as the number of known multiple-planet systems grows and our knowledge of them improves.

\subsection{Plan}

In Section 2, we present the sample of the 28 published nearby multiple-planet systems, including updated orbital parameters and radial velocities for ten of these systems. In Section 3, we present some empirical correlations among the properties of multiple-planet systems and difference between the distributions of orbital parameters among multiple-planet and single-planet systems. In Section 4, we briefly summarize our findings and discuss our principle conclusions.

\section{A SURVEY OF THE KNOWN MULTIPLE-PLANET SYSTEMS}

\subsection{New and Updated Multiplanet Systems}

We present updated velocities and orbital parameters for ten multiple-exoplanet systems in Tables 1 and 2 . In six systems, the updated orbits are substantially similar to the published orbits. In four cases there are major updates to previously published orbital parameters, which we detail below.

\subsubsection{HD 183263}

Marcy et al. (2005) announced an $M \sin i=3.7 M_{\text {Jup }}$ planet in a 634-day, eccentric $(e=0.4)$ orbit around HD 183263, a G2 IV star, and pointed out a strong residual linear trend of $32 \mathrm{~m} \mathrm{~s}^{-1} \mathrm{yr}^{-1}$. Wright et al. (2007) showed that by 2007 the residuals had significant curvature, but could not constrain the minimum mass of the outer companion. Since then, we have obtained observations on an additional six nights. These data have dramatically constrained the orbital fit to the outer planet, and we can now confidently measure the orbital elements of a 3-4 $M_{\text {Jub }}$ planet under the assumption that there is not a third companion contributing a detectable linear trend (see Wright et al. 2007 for a discussion of the difficulties of constraining planets with only partially observed orbits). HD $183263 c$ has an $8.4 \pm 0.3 \mathrm{yr}$ orbital period, orbits at $4.3 \pm 0.4 \mathrm{AU}$, and has $e=0.24 \pm 0.06$. We present the full set of newly determined orbital parameters in Table 1 , and the latest radial velocities in Table 2 and Figure 2. Figure 3 shows $\chi^{2}$ in $P-M \sin i$ space, demonstrating that, despite our having only one observed orbit, the minimum mass and orbital period of the planet are well constrained.

In Table 1, we report a stable solution near the $\chi^{2}$ minimum with errors estimated from the sample of stable orbits found through error bootstrapping (Wright et al. 2007). We tested each of the 100 bootstrapping trials for stability, and found a rough dividing line between stable and unstable solutions for this system such that orbital solutions with $e_{c}>10^{-4}\left(\left(P_{c} /\right.\right.$ days $)-$ $3000)$ are generally unstable. We have mapped this line into $P_{c}-m_{c} \sin i_{c}$ space in Figure 3, demonstrating that many solutions consistent with the data are, in fact, unstable. The true uncertainties are thus asymmetric about the nominal values because the $\chi^{2}$ minimum is so close to the boundary of stability.

For these long-term stability tests, we applied direct $n$-body integrations on each of the orbital solutions generated in the error bootstrapping. The radial velocity parameters were converted into initial conditions using a Jacobi coordinate system (Lee \& Peale 2003). Unless otherwise specified, we assumed edge-on, coplanar orbits. We held the stellar mass fixed, adopting values from Takeda et al. (2007). We integrated for at least $10^{8} \mathrm{yr}$ using the hybrid integrator in Mercury (Chambers 1999). For the majority of each integration, Mercury uses a mixed-variable symplectic integrator (Wisdom \& Holman 1991) with a time step equal to a hundredth of the Keplerian orbital period calculated at a semimajor axis equal to the pericenter distance of the closest planet. During close encounters, Mercury uses a Bulrich-Stoer integrator with an accuracy parameter of $10^{-10}$. We identified each set of initial conditions as an unstable system if: (1) two planets collide, (2) a planet is accreted onto the star (astrocentric distance less than $0.005 \mathrm{AU}$ ), or (3) a planet is ejected from the system (astrocentric distance exceeds $100 \mathrm{AU}$ ). We manually verified that for the vast majority of systems not identified as unstable, the final orbits are qualitatively similar to the initial conditions. 

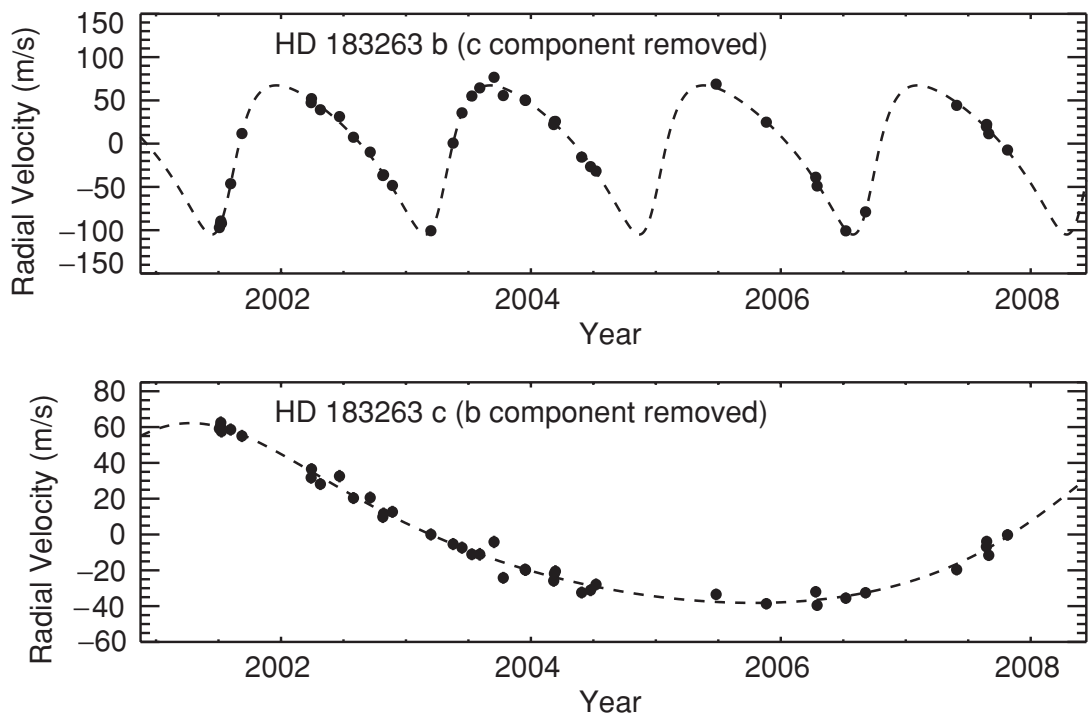

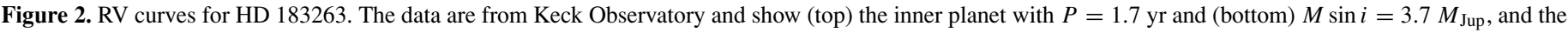
outer planet with $P \sim 8 \mathrm{yr}$ and $M \sin i=3.6 M_{\text {Jup }}$.

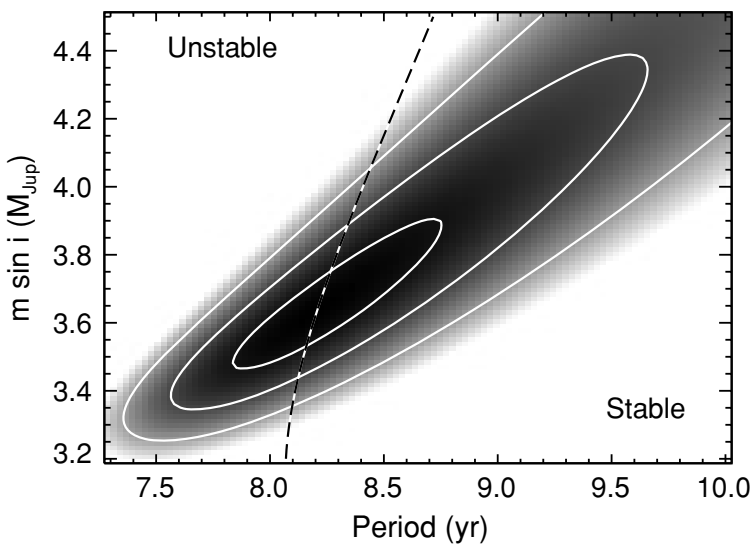

Figure 3. Contours of $\chi^{2}$ in $P_{c}-m_{c} \sin i$ space of best-fit orbits to the RV data of HD 183263 (Figure 2), with $\chi^{2}$ in grayscale. The solid contours mark the levels where $\chi^{2}$ increases by 1,4 , and 9 from the minimum. The dashed curve marks the approximate dividing line between stable and unstable orbital solutions, as determined by an ensemble of $n$-body integrations of the system assuming edge-on, coplanar orientations (see Figure 13 of Wright et al. 2007).

\subsubsection{HD 187123}

Butler et al. (1998) announced an $M \sin i=0.5 M_{\text {Jup }}$ planet in a three-day orbit around HD 187123, a close solar analog $\left(M_{*}=1.1 M_{\odot},[\mathrm{Fe} / \mathrm{H}]=+0.1, T_{\text {eff }}=5810\right)$. Wright et al. (2007) announced the existence of an outer companion with orbital period $>10 \mathrm{yr}$. At that time, the radial velocity history was too incomplete to fully determine the orbit, which had not yet closed. Wright et al. (2007) were nonetheless able to constrain the minimum mass of this "c" component to be planetary $\left(1.5 M_{\text {Jup }}<M \sin i<10 M_{\text {Jup }}\right)$. Since then, we have obtained additional observations of HD 187123, and have found that the orbit has closed just recently.

Assuming that there is no linear trend or detectable third planet in the system, the data constrain HD $187123 c$ to have $P=$ $10.4 \pm 1.2 \mathrm{yr}, e=0.25 \pm 0.03$, and $M \sin i=2.0 \pm 0.3 M_{\text {Jup }}$. We present the newly determined orbital parameters in Table 1 and the latest radial velocities in Table 2 and Figure 4. Figure 5 shows $\chi^{2}$ in $P-M \sin i$ space, demonstrating that, despite our having observed somewhat less than one full orbit, the minimum mass and orbital period of the planet are constrained to $\sim 20 \%$.

We have checked this orbit for long-term stability in the same manner as for HD 183263, and find that the range of solutions shown in Figure 5 are well within the stable regime.

\subsection{3. $H D 11964$}

HD 11964 (= GJ 81.1A) is a metal-rich $([\mathrm{Fe} / \mathrm{H}]=+0.12)$ slightly evolved ( 2 mag above the main sequence) $G$ star with a nearby (sep $\sim 30^{\prime \prime}$ ), K dwarf companion. Butler et al. (2006) announced the planet HD $11964 b$, a Jovian $\left(M \sin i=0.6 M_{\text {Jup }}\right)$ planet in a $5.5 \mathrm{yr}$, circular orbit, and noted a weak, residual trend in the velocities. An analysis by Wright et al. (2007) showed that a trend was probably not the proper interpretation of the residuals, and that they were consistent with a low amplitude $\left(K=5.6 \mathrm{~ms}^{-1}\right), 38 \mathrm{~d}$ signal (FAP $<2 \%$ ). Wright et al. (2007) cautioned that the low amplitude of this prospective $38 \mathrm{~d}$ planet meant that it would require more observations for confirmation, especially given the higher levels of jitter seen in subgiant stars (Wright 2005; Johnson et al. 2007b). Gregory (2007) also noted the $38 \mathrm{~d}$ period in a Bayesian periodgram of the published velocities (as well as a $360 \mathrm{~d}$ signal which is not apparent in the rereduced data presented here).

We have now obtained 24 additional observations of the star, and the $38 \mathrm{~d}$ signal has strengthened (FAP $<1 \%$ ), allowing us to confirm a low-mass $\left(M \sin i=23 M_{\oplus}\right)$ planet, HD $11964 c$, in a $37.9 \mathrm{~d}$ orbit. The low amplitude of the signal makes estimation of $e$ and $\omega$ difficult, but data are inconsistent with a circular orbit, and favor $e=0.3$.

Private communications from our group regarding the 38 d signal, which has been apparent, but not convincing, since 2005, has led to some confusion in the literature regarding the nomenclature of these planets (e.g., Raghavan et al. 2006). Here, we follow the convention that planet components are ordered by the date of a formal or a public announcement of their existence. We report the orbital parameters of the two-planet fit in Table 1, the radial velocities in Table 2, and we show the radial velocity curves in Figure 6. 

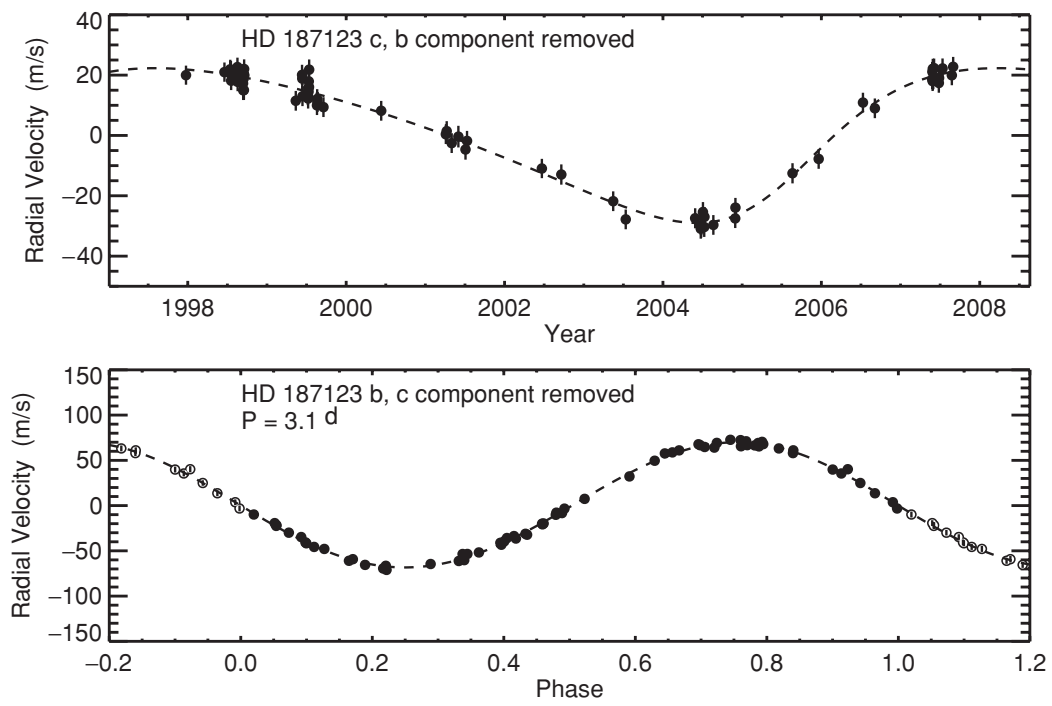

Figure 4. RV curves for HD 187123. The data are from Keck Observatory and show the inner planet (top) with $P=3.1 \mathrm{~d}$ and $M$ sin $i=0.5 M$ Jup, and the outer planet (bottom) with $P=10.7 \mathrm{yr}$ and $M \sin i=2 M_{\mathrm{Jup}}$.

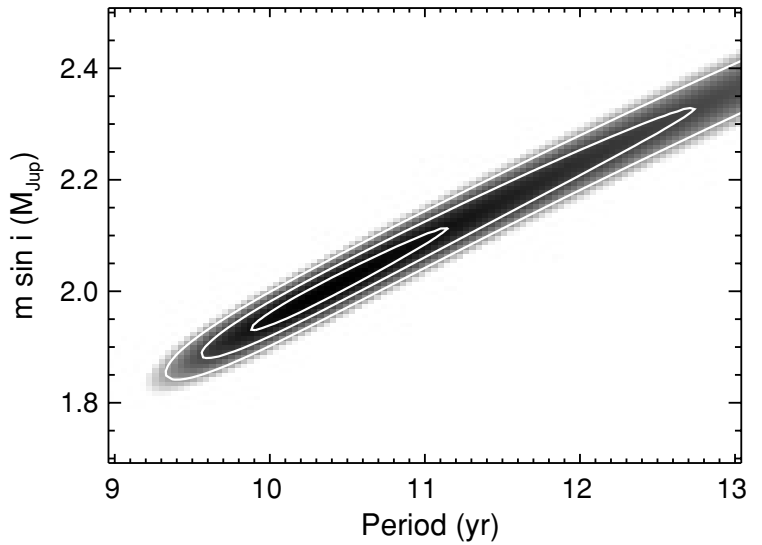

Figure 5. Contours of $\chi^{2}$ in $P_{c}-m_{c} \sin i_{c}$ space of best-fit orbits to the RV data of HD 187123 (Figure 4), with $\chi^{2}$ in grayscale (see Figure 15 of Wright et al. 2007). The solid contours mark the levels where $\chi^{2}$ increases by 1,4 , and 9 from the minimum. Although the orbit is still imprecise, the orbital period and minimum mass are now constrained to $\sim 20 \%$.

We have checked this orbit for long-term stability with the same $n$-body code as for HD 11964, and find that it is well within the stable regime.

\subsubsection{HD 217107}

Fischer et al. (1999) reported a $7.1 \mathrm{~d}, M \sin i=1.4 M_{\text {Jup }}$ planet orbiting HD 217107, and Fischer et al. (2001) described a linear trend superimposed on the Lick and Keck radial velocities. Vogt et al. (2005) updated the orbital fit, finding significant curvature in the residuals to the inner planet fit, and estimated its orbital period, though poorly constrained, to be $8.5 \mathrm{yr}$, with $M \sin i \sim 2 M_{\text {Jup }}$.

Since then, data collected at Lick and Keck continue to map out the orbit of the outer planet. Today, we can constrain the minimum mass and period to within $\sim 10 \%$ under the assumption that there are no additional planets in the system.

We present the newly determined orbital parameters in Table 1 and the latest radial velocities in Table 2 and Figure 7. Figure 8 shows $\chi^{2}$ in $P-M \sin i$ space, demonstrating that, despite our having observed somewhat less than one full orbit, the mass and orbital period of the planet are constrained to $\sim 10 \%$, at $P \sim 11.7$ yr and $M \sin i \sim 2.6 M_{\text {Jup }}$.

We have checked this orbit for long-term stability in the same manner as for HD 183263, and find that the range of solutions shown in Figure 8 are well within the stable regime.

\subsection{5. $47 U M a$}

Butler \& Marcy (1996) announced the existence of a 1090day planet orbiting $47 \mathrm{UMa}$ from data collected at Lick Observatory. After collecting an additional six years of data, Fischer et al. (2002a) announced the existence of a second, $0.46 M_{\text {Jup }}$ long period companion in a $\sim 2600$-day orbit. Naef et al. (2004) and Wittenmyer et al. (2007), using data from ELODIE and McDonald Observatory, respectively, have questioned the existence of $47 \mathrm{UMa} c$. Neither of the latter data sets, however, have both the precision and the duration to rule out the outer planet. The parameters quoted here are the literature values.

\subsection{The Current Sample}

We consider here the 28 known multiple-planet systems among the 205 known, normal exoplanet host stars within $200 \mathrm{pc}$. This is the sample of the Catalog of Nearby Exoplanets (CNE, Butler et al. 2006) ${ }^{8}$. The CNE employs a liberal upper mass limit in its definition of an exoplanet (any companion with $M \sin i<24 M_{\text {Jup }}$ ), but restricts itself to systems with high-quality radial velocity detections around the bright stars most amenable to confirmation and follow-up. This distance cutoff excludes the multiple planets orbiting the pulsar PSR 1257+12 (Konacki et al. 2003), and the Jupiter-Saturn analogs orbiting OGLE-2006-BLG-109 (Gaudi et al. 2008) detected by microlensing. We also exclude several speculative claims of additional planets around known exoplanet host stars, systems where second planets have very poorly constrained orbits, and two announced multiple planet systems (HD 47186 and HD 181433 , Bouchy et al. 2008) for which orbital parameters were not available at submission time.

We provide up-to-date fits with radial velocities as recent as 2008 June for those multiplanet systems with no significant

\footnotetext{
8 Available at http://exoplanets.org.
} 

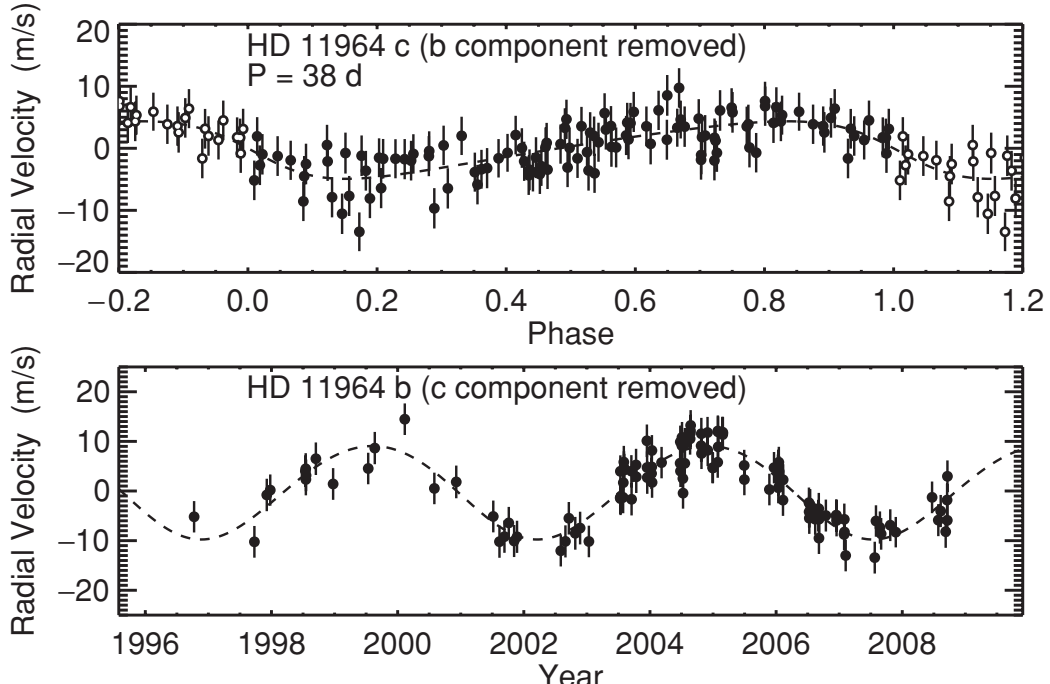

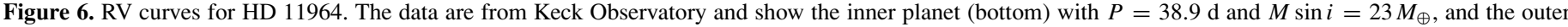
planet (top) with $P=5.5 \mathrm{yr}$ and $M \sin i=0.6 M_{\text {Jup. }}$.
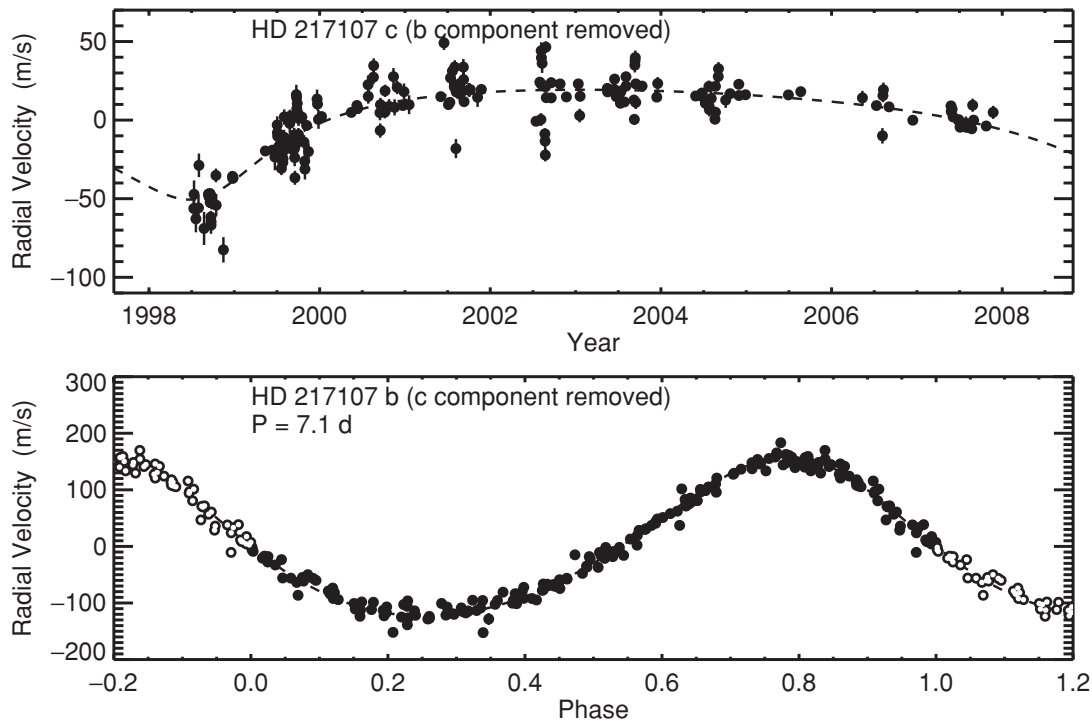

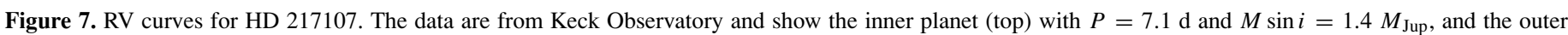
planet (bottom) with $P=11.6 \mathrm{yr}$ and $M \sin i=2.6 M_{\mathrm{Jup}}$.

planet-planet interactions (see Section 2.4), and for which we have Lick and Keck data from our planet search (see Butler et al. 2006, for details). We have employed a fitting algorithm which exploits linear parameters in the Kepler problem (Wright $\&$ Howard 2009) to efficiently search the high-dimensional $\chi^{2}$ space associated with multiple-planet systems. We have also updated some of our radial velocity data reduction procedures at Lick and Keck Observatories, including a small correction to our calculation of telescopic barycentric motion. The radial velocities presented here are thus more accurate and precise than our previously published velocities for these systems. The resulting best-fit orbital parameters and uncertainties supersede previously published parameters.

Table 1 contains measured properties and derived quantities for these 28 systems. Properties of the host stars can be found in Butler et al. (2006).

\subsection{The Multiplicity Rate}

In addition to these 28 systems, 36 single-planet systems are best fit with the addition of a linear trend (Butler et al. 2006). If we conservatively exclude the eight such cases in which the host star has a known stellar companion (determined from a survey of the literature including Eggenberger et al. 2007), then we are left with 28 apparently single-stars systems with an outer, potentially planetary companion. ${ }^{9}$ Of the 205 known nearby planetary systems then, $14 \%$ have multiple confirmed planets and another $14 \%$ show significant evidence of being multiple, meaning the true planet multiplicity rate may be $28 \%$ or higher. This is consistent with the estimate of Wright et al. (2007) and somewhat more conservative than the value of $\sim 50 \%$ in Fischer et al. (2001).

\subsection{Kinematic versus Dynamical Fits}

The radial velocity signature of multiple planets is significantly more complex than that of a single-planet system, and fitting such curves to observed radial velocity data requires care.

\footnotetext{
9 Our radial velocity analysis is sensitive to the presence of a second set of spectral lines, and we estimate that we can rule such a binary companion in a close orbit down to $\sim 0.1 M_{\odot}$ in most cases. Some contamination from as-yet undetected binary companions may still remain in the sample, however.
} 


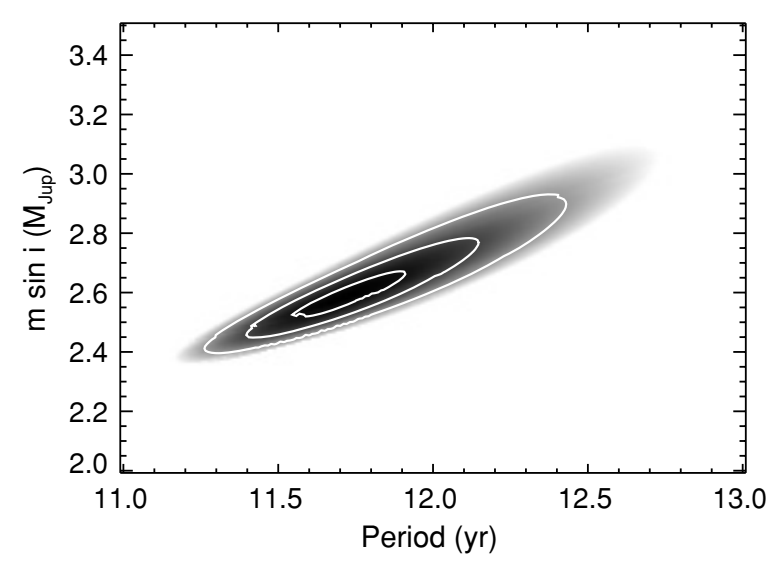

Figure 8. Contours of $\chi^{2}$ in $P_{c}-m_{c} \sin i_{c}$ space of best-fit orbits to the RV data of HD 217107 (Figure 7), with $\chi^{2}$ in grayscale (see Figure 15 of Wright et al. 2007). The solid contours mark the levels where $\chi^{2}$ increases by 1,4 , and 9 from the minimum. Although the orbit is still imprecise, the orbital period and minimum mass are now constrained to $\sim 10 \%$.

Each planet has five spectroscopic orbital parameters, most of which are neither orthogonal nor linear, so finding a global minimum in $\chi^{2}$ space becomes significantly more difficult as the number of planets grows. Efficient algorithms are necessary to conduct a thorough search (Wright \& Howard 2008).

Planets in mean motion resonances can be particularly difficult to identify from radial velocity curves because of degeneracies among the best-fit orbital parameters. Care must also be taken not to confuse weak signals resulting from aliasing of orbital periods with the observing window function with genuine planet detections (Fischer et al. 2008; Tinney et al. 2006).

Most importantly, interactions between planets may require consideration. In many cases, these planet-planet interactions are sufficiently weak that they can be ignored, and the resulting radial velocity signal is simply the linear superposition of multiple Keplerian radial velocity curves (a "Keplerian" or a "kinematic" fit).

When these interactions are important, however, both shortterm and long-term numerical $n$-body integrations of the physical system must be performed. In the short term, these interactions can cause detectable variations in the orbits of the planets (Rivera et al. 2005; Fischer et al. 2008). In these cases, a set of constant Keplerian orbital elements is insufficient to model the observed radial velocities, and a proper fit must be driven by an $n$-body code (a "Newtonian" or a "dynamical" fit).

In some cases, even good Newtonian fits to the data may yield orbital parameters for planets which, while stable for the duration of the observations, are not stable on timescales comparable to the age of the planetary system. Thus, long-term stability is an additional constraint that multiplanet fits must satisfy.

The new fits listed in Table 1 are all Keplerian fits which have been confirmed stable, with the $n$-body code described in Section 2.1.2. The compiled literature fits are a mixture of Keplerian and Newtonian fits, and in some cases a more sophisticated Newtonian fit may be superior to the published one. For instance, the orbits of the planets in the HD 74156 system reported by Bean et al. (2008) are apparently unstable on $10^{5} \mathrm{yr}$ timescales, but an orbit with similar parameters is stable (Barnes et al. 2008). Our conclusions on the statistical properties of planets are insensitive to these details, but detailed work on planet-planet interactions and resonant dynamics should employ the published RV data directly, rather than the orbital elements presented here.

\section{STATISTICS OF MULTIPLE-PLANET SYSTEMS}

\subsection{Semimajor Axis Distributions}

Figure 9 shows the distribution of semimajor axes ${ }^{10}$ for multiplanet and apparently single-planet systems. The semimajor axes of multiplanet systems appear to show some significant departures from the single-planet systems. Most strikingly, the pileup of hot Jupiter planets between $0.03 \mathrm{AU}$ and $0.07 \mathrm{AU}$ and the jump at $1 \mathrm{AU}$ observed in the distribution for single-planet systems are both absent from the corresponding distribution for multiplanet systems, which appears rather uniform.

It thus appears that planets in multiple systems are not consistent with having been drawn randomly from the population of apparently single systems. To provide a numerical measure of the difference between the two distributions, we created the cumulative distribution function (CDF) of the semimajor axis distribution (with an upper limit of $13 M_{\mathrm{Jup}}$ ) and applied a Monte Carlo Kolmogorov-Smirnov (K-S) test for a difference in distribution. The $\mathrm{K}-\mathrm{S}$ test uses the two sided $D$-statistic, defined as the maximum difference between the two CDF curves. The Appendix contains a complete description of our Monte Carlo K-S procedure.

Figure 10 shows the CDF for these two distributions. A K-S test of the two distributions shows that we can reject the null hypothesis that the two samples were drawn from the same distribution with $90 \%$ confidence.

This is somewhat surprising, since presumably many apparently single-planet systems have as-yet undetected low-mass planets and are, in fact, multiple systems. Presumably, then the difference is due to the presence of multiple giant planets within 5 AU.

In particular, close-in planets $(a<0.07 \mathrm{AU})$ are not as common in multiplanet systems as in apparently single-planet systems. Among the 88 single planets having $a<1 \mathrm{AU}, 38$ of them (43\%) have $a<0.07$ AU. A similar rate for the 39 planets in multiple systems with $a<1$ AU would yield 15 planets within $0.07 \mathrm{AU}$, but only seven are known.

The $1 \mathrm{AU}$ jump in the single-planet sample may relate to the location of the "ice line" beyond which ices can participate in planet formation in the protoplanetary nebula. If this peak therefore represents planets which have not experienced significant migration, then the lack of such a peak in the multiple-planet distribution suggests that planets in multiple-planet systems generally undergo more significant migration.

Alternatively, the $1 \mathrm{AU}$ jump may be indicative of a pileup of planets at the typical orbital distance within which migration becomes inefficient due to either the presence of the ice line or a "dead zone" near 1 AU (e.g., Ida \& Lin 2008). For instance, in a model where planet migration is driven by planetesimal scattering, the disk surface density of planetesimals is large enough to drive migration beyond the ice line, but not inside it.

\section{2. $M \sin i$}

The distribution of minimum masses $(M \sin i)$ of planets in multiple-planet systems is shown in Figure 11, along with

\footnotetext{
${ }^{10}$ Following Butler et al. (2006), we calculate semimajor axes from the measured orbital periods using Newton's version of Kepler's Third Law; the major source of uncertainty in $a$ is usually the host star's mass.
} 


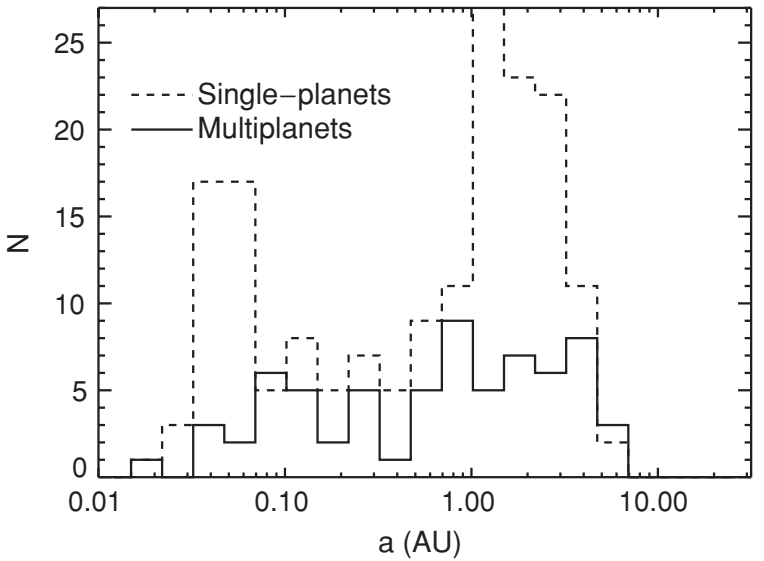

Figure 9. Distribution of semimajor axes of exoplanets for multiple-planet systems (solid) and apparently single systems (dashed). Note the enhanced frequency of hot Jupiters and the jump in abundance beyond $1 \mathrm{AU}$ in the singleplanet systems.

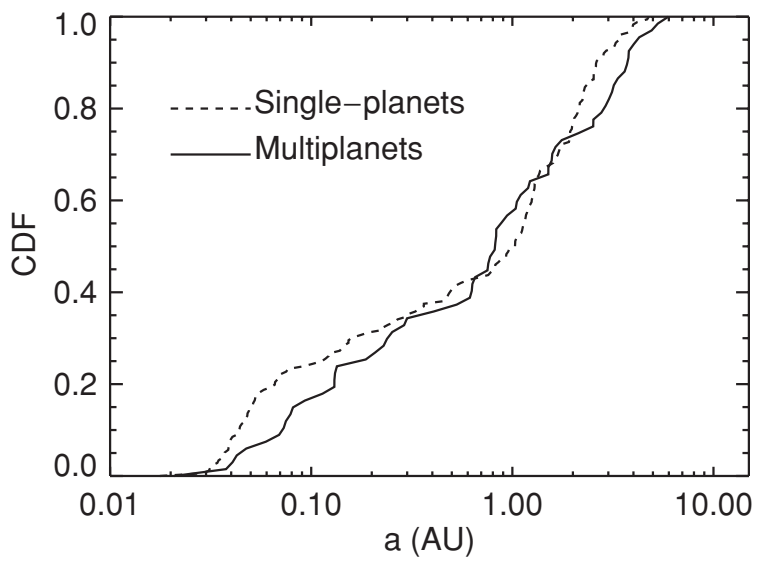

Figure 10. CDF of semimajor axis for known multiplanet systems (solid) and apparently single systems (dashed).

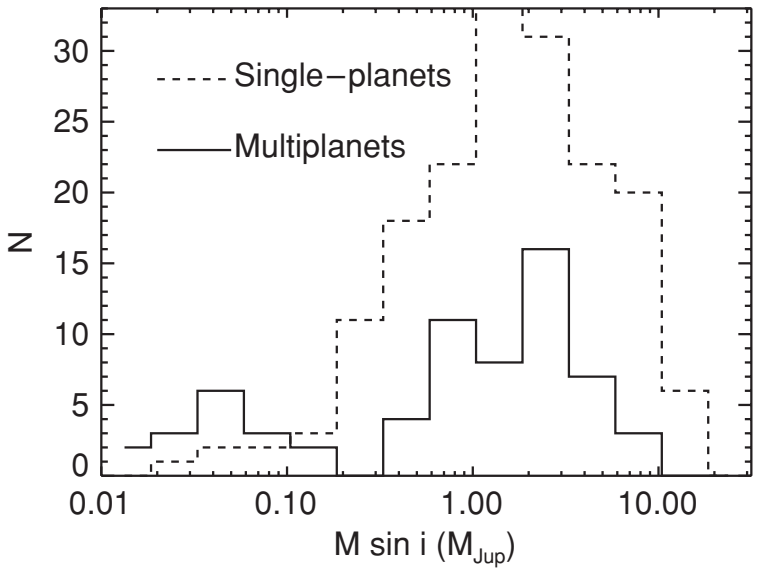

Figure 11. Distribution of $M \sin i$ of exoplanets, with an upper limit of $13 M_{\text {Jup }}$ for known multiple-planet systems (solid) and apparently single systems (dashed).

that for single planets. Multiplanet systems exhibit an apparent overabundance of planets with $M \sin i$ between 0.01 and $0.2 M_{\text {Jup }}$, but this may be amplified by a selection effect. When we find a planet around a star we tend to observe that star more frequently-making it more likely that we will find another planet that was not detectable beforehand. This appears to explain the detection of very low-mass planets around $55 \mathrm{Cnc}$, GJ

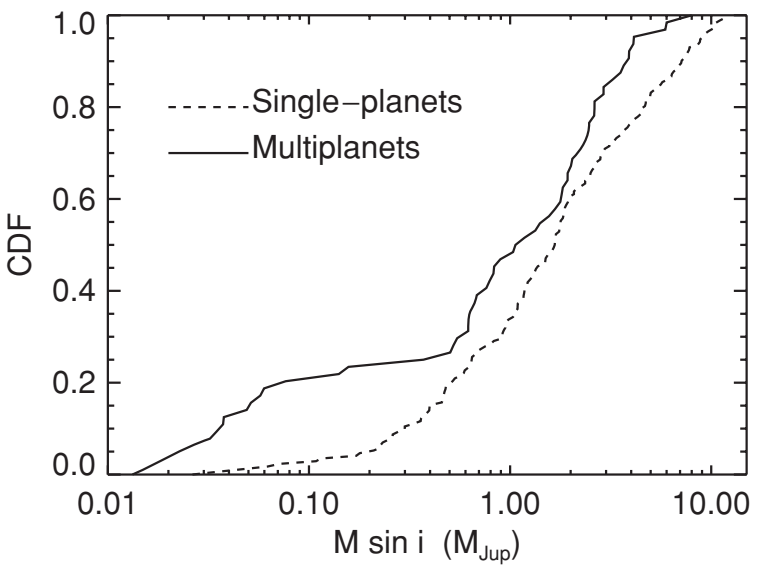

Figure 12. CDF of $M \sin i$ for known multiplanet systems (solid) and apparently single systems (dashed). Note the enhanced frequency of known low-mass planets in multiplanet systems.

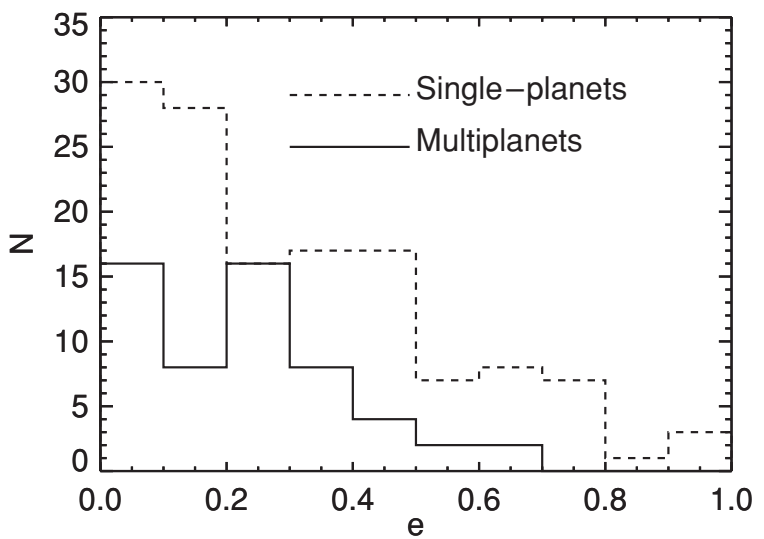

Figure 13. Distribution of eccentricities of exoplanets for known multiple-planet systems (solid) and apparently single-planet systems (dashed). Note the high eccentricity orbits, $e>0.6$ occur predominantly in single planets.

876, and $\mu$ Ara. In these systems, more massive planets were known in advance, and an especially large number of observations were made to refine their orbits. The lowest-mass planets were found in the course of these detailed observations.

The $M \sin i$ CDF of single-planet systems (Figure 12) is relatively featureless with the $\operatorname{logarithmic} M \sin i$ axis, but the CDF of multiplanet systems deviates markedly from its singleplanet counterpart. We calculate $D^{+}=1.36$ and $D^{-}=-0.089$, so we find $p\left(D^{+}\right)=1.8 \%$, and so we can reject the null hypothesis that our samples are from a common distribution with $>95 \%$ confidence.

\subsection{Eccentricity}

The distribution of eccentricities for single and multiplanet systems are shown in Figure 13. Note that we have excluded planets with $a<0.1 \mathrm{AU}$ from consideration here to remove the effects of tidal circularization on the analysis. Of the planets selected, both single and multiplanet systems exhibit a wide range of eccentricities from 0.0 to 0.8 . The mean and standard deviation for single planets are 0.30 and 0.24 , respectively, and 0.22 and 0.17 for multiplanet systems. Also, 11 single planets have eccentricities above 0.7 ( $7 \%$ of this sample), but none of the multiplanet systems has an eccentricity above 0.7 .

To provide a numerical measure of the significance of the difference between the two distributions, we computed the 


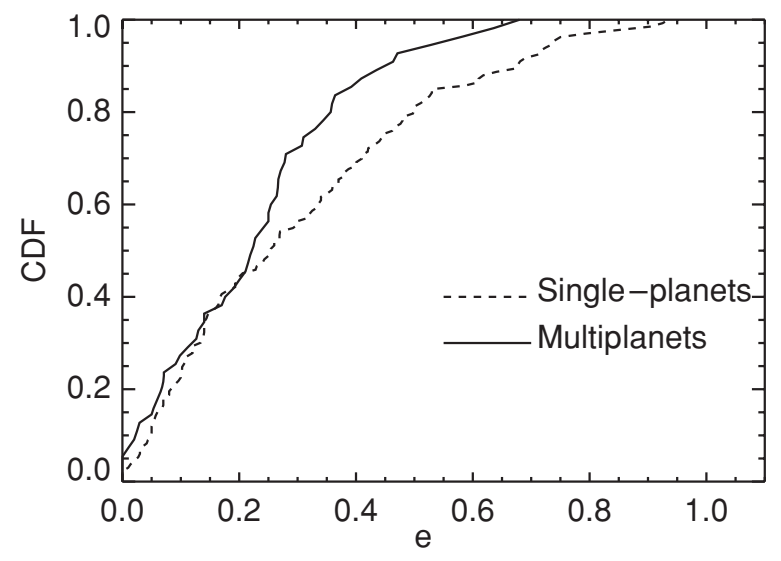

Figure 14. CDF of eccentricity for multiplanet systems (solid) and apparently single systems (dashed). The tidally circularized hot Jupiters have been removed. Note that the highest eccentricities, $e>0.6$ occur predominantly in the singleplanet systems.

cumulative distribution function of the eccentricities and applied a Monte Carlo K-S test for difference of distribution (Figure 14). The eccentricity CDF for multiplanet systems is greater than the eccentricity CDF for single-planet systems at any given eccentricity, suggesting that multiplanets systems have systematically lower eccentricity. The K-S statistics are $D^{+}=1.34$ and $D^{-}=0.265$, and $p\left(D^{+}\right)=<1 \%$, so we reject the null hypothesis that our samples are from a common distribution with over $99 \%$ confidence. Thus, it appears that the known multiplanet systems have systematically lower eccentricities. Selection effects in eccentricity do not significantly affect the delectability of planets for $e<0.7$ (Cumming et al. 2008).

It is surprising that multiplanet systems have lower orbital eccentricities, as mutual interactions between giant planets might be expected to excite eccentricities. The lack of very high eccentricities in multiplanet systems may be partially explained by the additional constraint in multiplanet systems of orbital stability, which favors low-eccentricity orbits. Conversely, some single-planet systems may exhibit high eccentricities as a result of a series of ejections of former members from the system. Both factors can be at play simultaneously: Ford et al. (2005) explain the observed eccentricities of the planets in the $v$ Andromedae system as the end result of the ejection of a hypothesized fourth planet from the system.

\subsection{Metallicity}

Fischer \& Valenti (2005), using uniformly calculated metallicities from Valenti \& Fischer (2005), found that the 14 multiple-planet systems then known had a somewhat higher average metallicity than single-planet systems $(+0.18$ versus +0.14 ), suggesting that metallicity traces multiplicity in planets even more strongly than it traces single-planet occurrence (see also Santos et al. 2001). They suggested that further discoveries of multiplanet systems could confirm this trend. Using the metallicities compiled ${ }^{11}$ in the $\mathrm{CNE}$, we find that single and multiplanet systems have mean $[\mathrm{Fe} / \mathrm{H}]$ values of +0.10 and +0.10 , respectively, although the median values are +0.15 and +0.18 , still showing some evidence of the disparity.

If we include systems showing long-term RV trends (not including known binaries) among the multiple-planet systems,

\footnotetext{
11 In most cases, the ultimate origin of these metallicites is Valenti \& Fischer
} (2005).

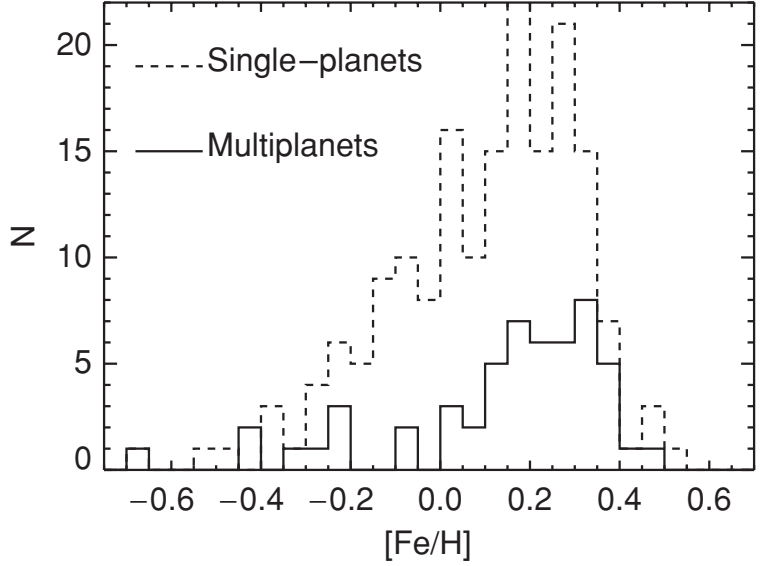

Figure 15. Distribution of $[\mathrm{Fe} / \mathrm{H}]$ for exoplanet-bearing stars harboring likely multiple-planet systems (including single-planet systems with long-term RV trends; solid line) and apparently single systems (dashed). The median $[\mathrm{Fe} / \mathrm{H}]$ for known multiplanet systems, +0.18 , is higher than that for the single-planet systems, +0.14 . The multiplanet system HD 155358 has the lowest $[\mathrm{Fe} / \mathrm{H}]$ of any system, with $[\mathrm{Fe} / \mathrm{H}]=-0.68$.

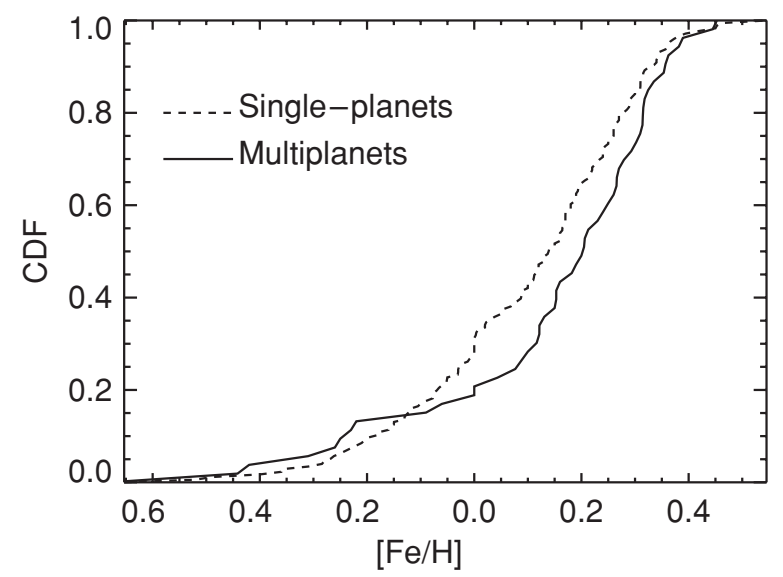

Figure 16. $\mathrm{CDF}$ of $[\mathrm{Fe} / \mathrm{H}]$ for known multiple-planet systems (solid) and apparently single systems (dashed). The CDF shows that the metallicity of the multiplanet systems is consistently higher than that of the single-planet systems.

however, the difference becomes slightly stronger. Such systems have a mean $[\mathrm{Fe} / \mathrm{H}]$ value of +0.20 , bringing the average for apparent multiple systems overall up to $[\mathrm{Fe} / \mathrm{H}]=+0.15$.

We plot the two distributions in Figure 15. To test whether these two distributions differ significantly, we have performed a $\mathrm{K}-\mathrm{S}$ test, as shown in Figure 16. A K-S test rejects, with $97 \%$ confidence, the null hypothesis that the apparently multiplanet systems (including systems with trends) have metallicities drawn from the same distribution as the single-planet systems. It appears that metallicity traces not only planet occurrence rate, but multiplicity among planet-bearing stars as well.

\subsection{Stellar Mass}

The distributions of stellar mass for stars hosting single planets and stars hosting multiplanet systems are shown in Figure 17. Multiplanet systems have a mean stellar mass of $1.1 M_{\odot}$ and single-planet systems have a mean mass of $1.13 M_{\odot}$. $\mathrm{K}-\mathrm{S}$ tests of the histogram in Figure 17 and of the corresponding CDF show no significant difference between the stellar masses of single and multiplanet systems. Unlike metallicity, stellar mass does not seem to strongly trace multiplicity. 


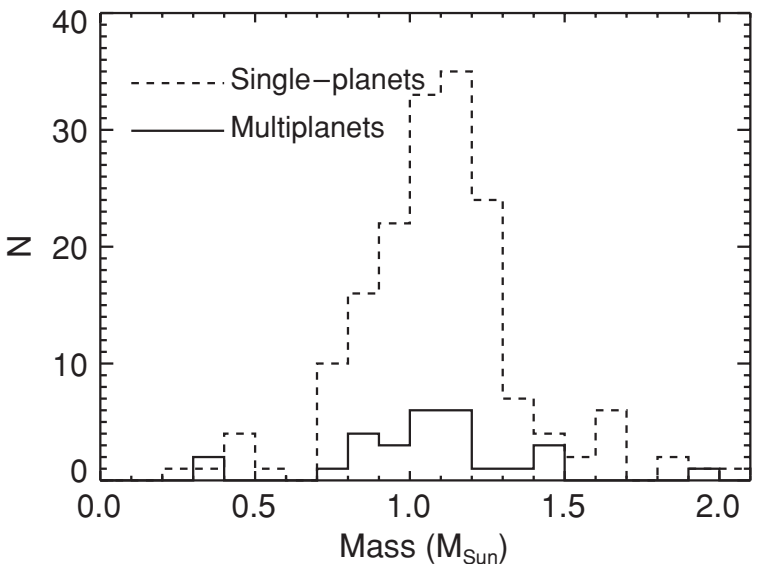

Figure 17. Mass distribution of exoplanet-bearing stars for known multipleplanet systems (solid) and apparently single systems (dashed). There is no significant difference between the stellar masses of the single and multiplanet systems.

\subsection{Multiplicity versus Stellar Mass}

Although there is no strong stellar-mass-multiplicity relation, there may be an emerging trend regarding the $M$ dwarfs. Among the eight known M dwarf exoplanet hosts, two are well-characterized multiple-planet systems (GJ 876 and GJ 581) and two others (GJ 317 and GJ 849) show a trend in the singleplanet velocity residuals. If we attribute these trends to planets and not undetected stellar or brown dwarf companions, then the total, true multiple-planet rate is at least $50 \%$, which is higher than the $28 \%$ similarly calculated for the entire CNE sample in Section 2.3. It appears that planets around M dwarfs may be found preferentially in multiplanet systems rather than singly. This trend, if it is not simply Poisson noise and should hold up as more $\mathbf{M}$ dwarf systems are discovered, is especially surprising since $\mathrm{M}$ dwarfs have a lower-than-average planet occurrence rate (Endl et al. 2006; Johnson et al. 2007a; Mayor et al. 2009).

There may be subtle observational selection effects at work here, however. If $\mathbf{M}$ dwarfs in general have lower mass planets than F-K stars (and thus require more observations before publication), then we may simply be seeing the already-documented increase in planet occurrence rate amongst low-mass planets. The ongoing RV M dwarf surveys will improve the statistics of these systems, which should help illuminate if the effect is due to selection effects, small numbers, or astrophysics.

\subsection{Eccentricity versus $M \sin i$}

Consider the plot of eccentricity versus $M \sin i$ of planets with $a>0.1 \mathrm{AU}$, including both multi- and single-planet systems (Figure 18). We have excluded planets within 0.1 AU to remove the effects of tidal circularization on our analysis. Planets with minimum mass below $1.0 M_{\text {Jup }}$ have a mean eccentricity of 0.19 , while planets above this threshold have a mean eccentricity of 0.34 .

Figure 19 shows the strong dichotomy between the eccentricity distributions of super- and sub-Jupiters: the eccentricity of sub-Jupiters peaks at $e<0.1$, while the eccentricity of super-Jupiters is distributed broadly from $0.0<e<0.6$. Figure 20 shows that a $\mathrm{K}-\mathrm{S}$ statistic bears out this difference: for these two populations, $D^{+}=2.06$ and $D^{-}=-.034$, yielding $p\left(D^{+}\right)<0.1 \%$, so we reject the null hypothesis that the samples are drawn from the same distribution, and conclude that the apparent difference is not a chance result of small-number statistics.

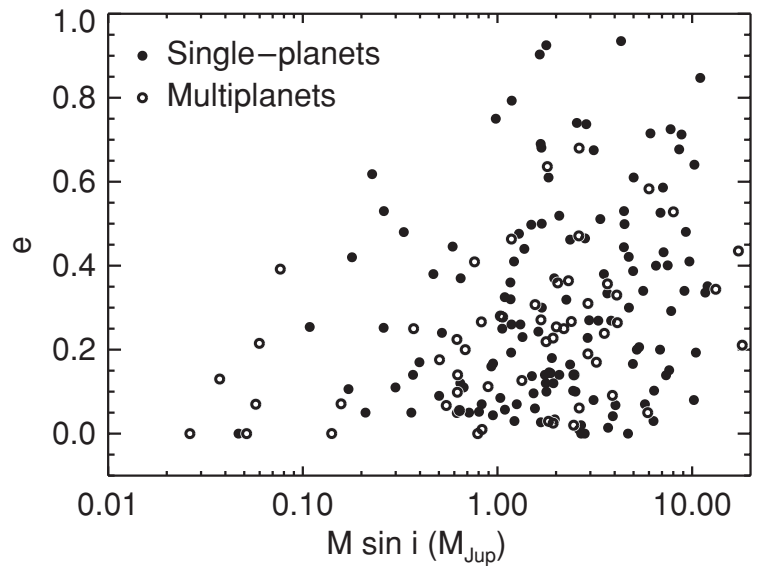

Figure 18. Plot of eccentricity vs. $M \sin i$ for planets with $a>0.1$ AU to avoid contamination from tidal circularization. Filled circles are single-planet systems, and open circles represent multiplanet systems. There is a slight sense of increase in the upper envelope in the range $0.05-1.0 \mathrm{M}_{\mathrm{Jup}}$.

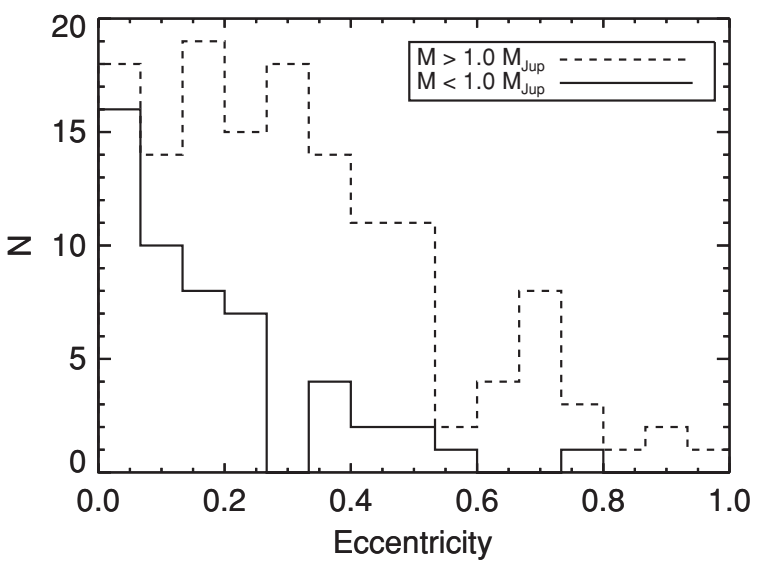

Figure 19. Distribution of eccentricities of exoplanets with $M \sin i<1.0 M_{\text {Jup }}$ (solid) and $M \sin i>1.0 M_{\text {Jup }}$ (dashed). The tidally circularized hot Jupiters have been removed. Note that the eccentricity of planets of minimum mass $<1.0 M_{\text {Jup }}$ peaks at eccentricity $<0.2$, while the eccentricities $e$ of planets of minimum mass $>1.0 M_{\text {Jup }}$ are distributed broadly from $0.0<e<0.6$.

This correlation between eccentricity and planet minimum mass provides a valuable clue about the origins of eccentricities. One possibility is that conditions that encourage the formation of planets with $M \sin i>1 M_{\text {Jup }}$ may contribute to greater eccentricity pumping as well. If so, then these would need to be strong effects as more massive planets are relatively more difficult to perturb. Also possibly relevant is the work of Goldreich \& Sari (2003), who describe a mechanism for modest eccentricity pumping of a planet through interactions with a protoplanetary disk that should be more efficient for more massive planets. This mechanism could create a "seed" eccentricity preferentially in massive planets, which would grow through planet-planet interactions. Alternatively, it is possible that significant eccentricities are the norm for all systems shortly after the dissipation of the protoplanetary disk triggers strong planet-planet interactions (e.g., Ford 2006), and that circularization through dynamical friction with planetesimals is more efficient for planets with $M<1.0 M_{\text {Jup }}$. Another possibility, that this is the signature of eccentricity pumping by planet-planet scattering, is discussed in Section 3.10. 


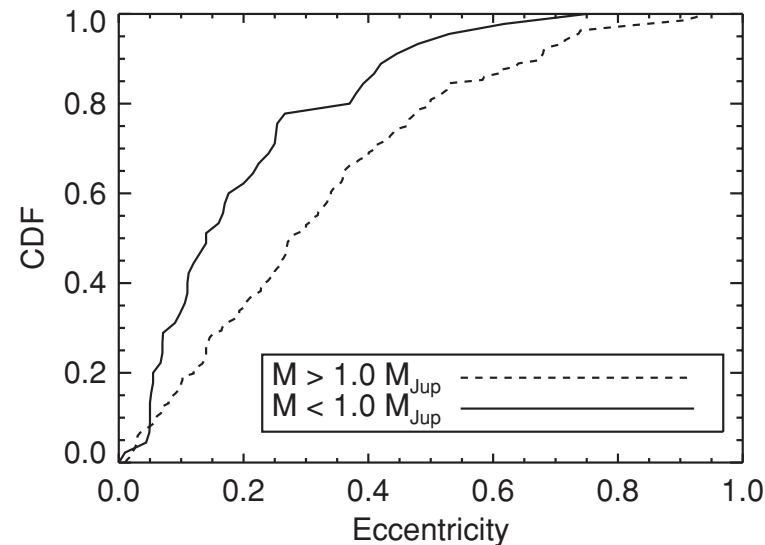

Figure 20. CDF of eccentricity for planets of minimum mass $<1.0 M_{\text {Jup }}$ (solid line) and minimum mass $>1.0 \mathrm{M}_{\mathrm{Jup}}$ (dashed line). The tidally circularized hot Jupiters have been removed. Note that the CDF for the light planets rises steeply from eccentricity 0.0 to 0.2 , while the CDF for the heavy planets rises with a nearly uniform slope from eccentricity 0.0 to 0.6 .

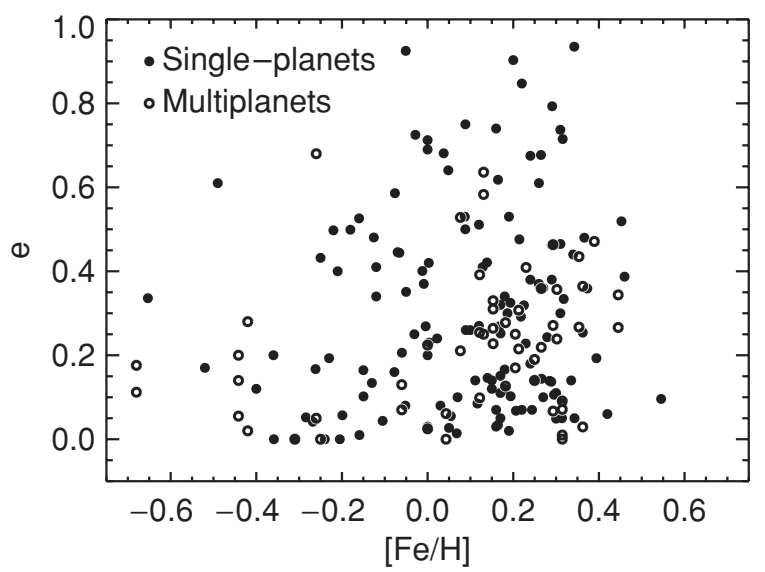

Figure 21. Plot of eccentricity vs. $[\mathrm{Fe} / \mathrm{H}]$ for planets with $a>0.1$ AU to remove bias caused by tidal circularization. Filled circles are single-planet systems, and open circles represent multiplanet systems. No correlation is apparent.

\subsection{Eccentricity versus Metallicity}

We plot eccentricity versus metallicity $([\mathrm{Fe} / \mathrm{H}])$ for planets with $a>0.1 \mathrm{AU}$, including both multi and single-planet systems (Figure 21). There appears to be no significant correlation between $[\mathrm{Fe} / \mathrm{H}]$ and eccentricities. While the systems with $[\mathrm{Fe} / \mathrm{H}]<-0.2$ appear to have lower eccentricities than those with $[\mathrm{Fe} / \mathrm{H}]>-0.2$, this may be due to the small number of such systems.

\subsection{Eccentricity versus Semimajor Axis}

Figure 22 shows the eccentricity versus semimajor axis for all planetary systems. There is a clear paucity of planets with small $a$ and large $e$, as expected from the effects of tidal circularization (Rasio et al. 1996; Ford et al. 1999).

Planets from $0.5 \mathrm{AU}$ to about $3 \mathrm{AU}$ have the widest range of eccentricities, and beyond $3 \mathrm{AU}$ there may be a paucity of planets with $e>0.6$. This could easily be due to observational biases: planets beyond $3 \mathrm{AU}$ have such long orbital periods that only a small number of orbits have necessarily been observed. In cases with fewer than two complete orbits, the radial velocity signature of any additional exterior planets with orbital periods longer than the span of the observations can sometimes be absorbed into the eccentricity term of the orbital solution of the inner

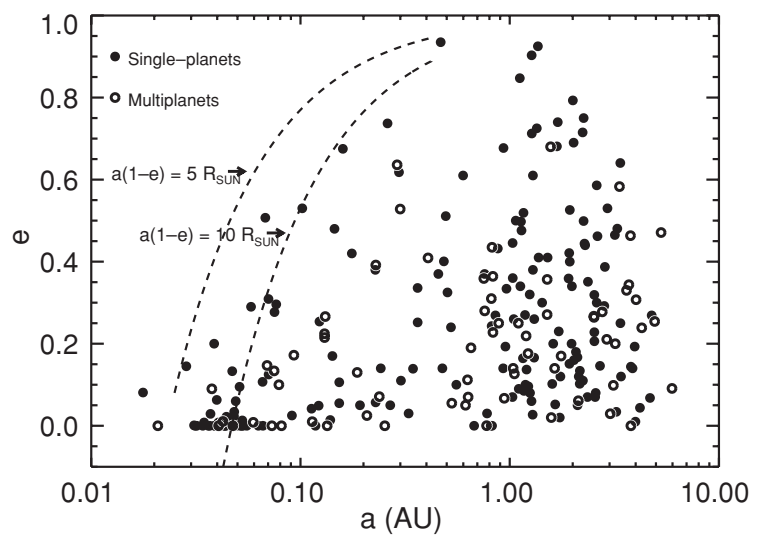

Figure 22. Plot of eccentricity vs. semimajor axis. Filled circles are single-planet systems, and open circles represent multiplanet systems. Note the increasing upper envelope from 0.01 to $0.5 \mathrm{AU}$. We have drawn the curves representing periastron passage distances of $a(1-e)=5 R_{\odot}$ and $a(1-e)=10 R_{\odot}$ with dashed lines for reference.

planet. Further, since an $e>0.6$ planet spends a small fraction of its orbit near periastron, where its velocity signal is largest, such a planet may not reveal itself until it has completed nearly an entire orbit. The discovery of more long-period planets and the observation of more complete orbits will help reveal the true eccentricity distribution of planets with $a>3 \mathrm{AU}$.

\subsection{Ratio of Escape Speeds versus Eccentricity}

Some theoretical models of the evolution of eccentricity through planet-planet scattering focus on the parameter $\theta$, defined $^{12}$ as the ratio of the escape speed from the planet to that of the planetary system (Rasio 2008),

$$
\theta^{2} \equiv\left(\frac{G M}{R_{p}}\right)\left(\frac{r}{G M_{*}}\right),
$$

where $M$ is the mass of the planet, $R_{p}$ its radius, $r$ its orbital distance, and $M_{*}$ is the mass of the host star. Because we do not have exact masses or radii for many of the exoplanets in our sample, and because the following analysis is rather insensitive to the exact values of those quantities, here we approximate

$$
\theta^{2} \sin i=10\left(\frac{M \sin i}{M_{\mathrm{Jup}}}\right)\left(\frac{M_{\odot}}{M_{*}}\right)\left(\frac{R_{\mathrm{Jup}}}{R_{p}}\right)\left(\frac{a(1+e)}{5 \mathrm{AU}}\right),
$$

and crudely estimate exoplanetary radii from the assumption that exoplanets below the mass of Jupiter have similar mean densities,

$$
\left(\frac{R_{p}}{R_{\mathrm{Jup}}}\right)^{3}=\left\{\begin{array}{cl}
\frac{M \sin i}{M_{\mathrm{Jup}}} & M \sin i<M_{\mathrm{Jup}} \\
1 & M \sin i>M_{\mathrm{Jup}}
\end{array}\right.
$$

When $\theta \gg 1$, a planet can efficiently eject bodies during close encounters, and when $\theta<1$ collisions are more frequent. Figure 23 shows the distribution of $\theta^{2} \sin i$ versus eccentricity for single planets and those in multiple-planet systems. Consistent with Ford \& Rasio (2008), Figure 23 shows that planets that scatter planets and planetesimals efficiently (i.e., those with $\theta \gg 1$ ) have a wider range of eccentricities than inefficient scatterers. This is consistent with the hypothesis that planet-planet and planet-planetesimal scattering is a dominant mechanism for the excitation of exoplanetary eccentricites.

\footnotetext{
12 Some authors refer to this quantity as the Safronov number.
} 


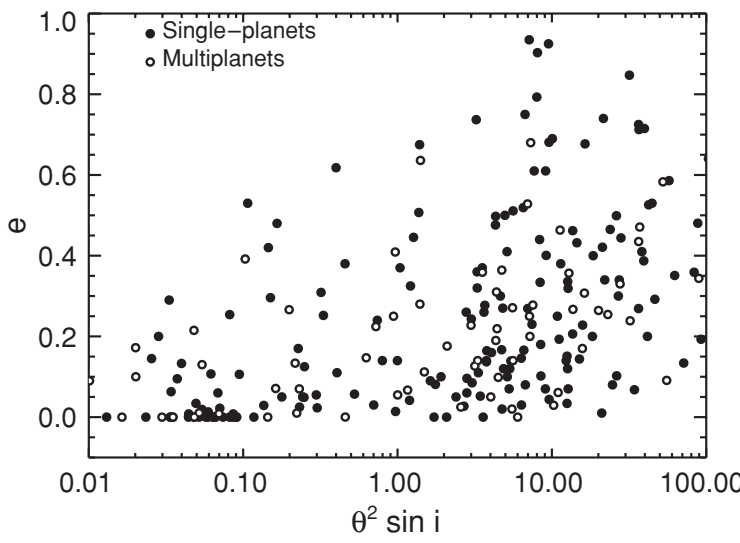

Figure 23. Eccentricity vs. the ratio of the escape velocity from the planet to the escape velocity of the $\operatorname{star}\left(\theta^{2}\right)$.

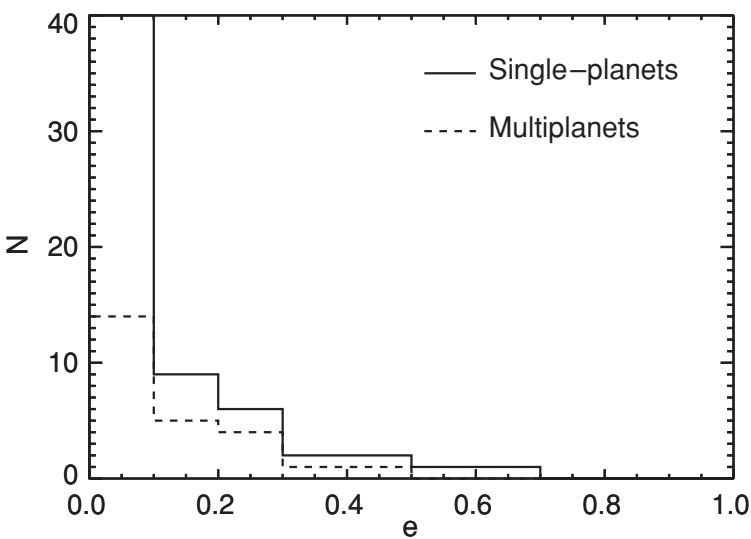

Figure 24. Distribution of eccentricites for single- and multiple-planet systems with $\theta<1$.

Because $\theta^{2} \propto M \sin i$, these results are consistent with Section 3.7 where we showed that the eccentricity distribution of planets with $M \sin i<1 M_{\text {Jup }}$ is peaked at very small $e$ : the eccentricity dichotomy between high- and low-mass planets may be related to the ability of high-mass planets to more efficiently scatter planets and planetesimals.

If we divide the sample into those planets with $\theta<1$ and those with $\theta>1$, we can examine whether the eccentricity distributions of efficient scatterers in single and multiplanet systems differ from one another. Figures 24 and 25 show that inefficient scatterers in single and multiplanet systems have very similar eccentricity distributions. Interestingly, Figures 26 and 27 show that efficient scatterers (those for which $\theta>1$ ) in multiplanet systems appear to have higher eccentricities than those in single-planet systems. A K-S test for these two populations gives $D^{+}=1.36$ and $D^{-}=0.11$, yielding $p\left(D^{+}\right)=2 \%$, showing that it is unlikely that these samples are drawn from the same distributions. This is despite the overall tendency of multiplanet systems to have lower eccentricities (see Section 3.3.)

\subsection{Metallicity and Stellar Mass versus $M \sin i$}

Since metallicity and stellar mass both correlate with the occurrence rate of planets, it is reasonable to check to see if either also correlates with minimum planet mass. In Figures 28 and 29, we plot $M \sin i$ versus these two quantities. Neither figure shows a strong correlation. There is a dearth of low-mass planets found around high-mass stars, but this may simply be

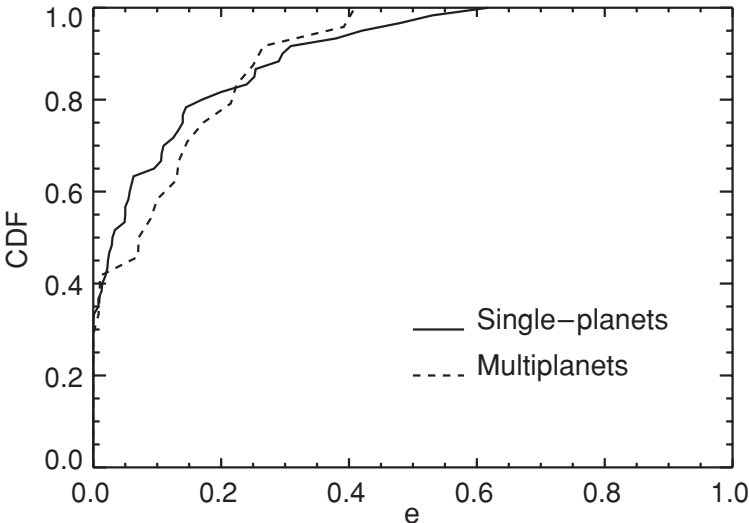

Figure 25. CDF for eccentricity for $\theta<1$ planets in single and multiplanet systems. The eccentricity distributions for these inefficient scatterers do not differ significantly.

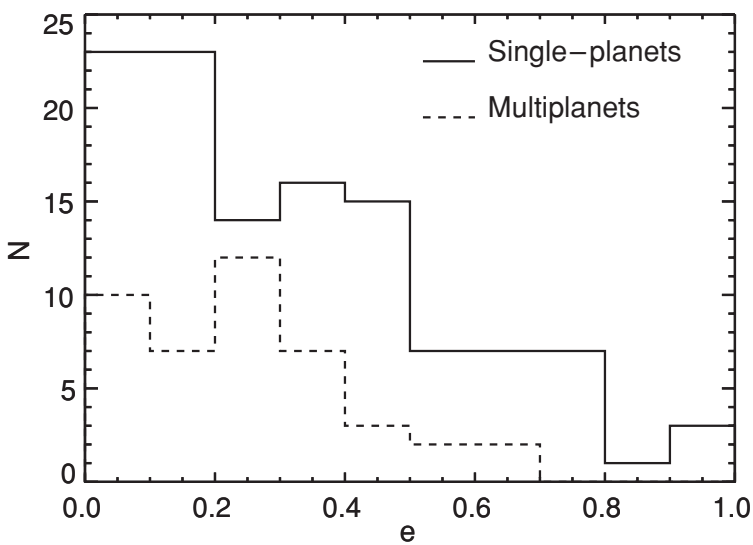

Figure 26. Distribution of eccentricites for single- and multiple-planet systems with $\theta>1$.

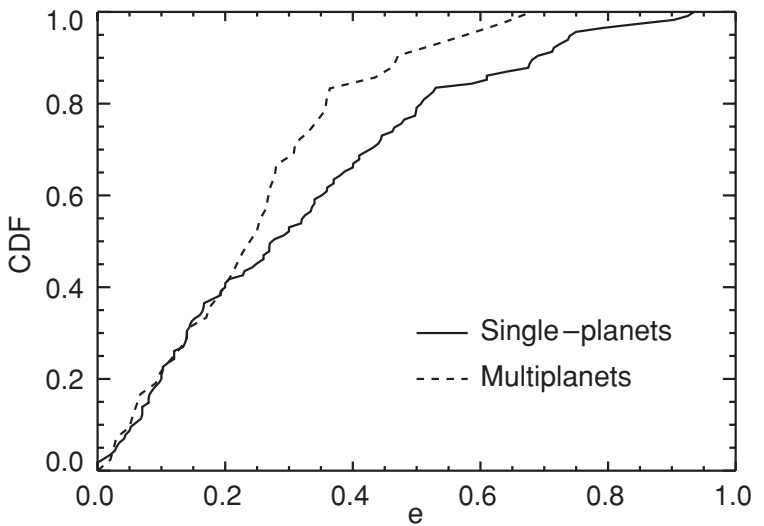

Figure 27. CDF for eccentricity for $\theta>1$ planets in single and multiplanet systems. The eccentricity distributions for these efficient scatterers differ significantly. It appears that efficient scatterers in multiplanet systems display larger eccentricities than those in single-planet systems.

an observational artifact, as such planets would have a lower reflex amplitude and so be more difficult to detect. Likewise, the typical minimum mass of planets orbiting $\mathrm{M}$ dwarf stars appears to be lower than that around solar mass stars, but this may simply be an artifact of the fact that these low-mass planets are more detectable around $\mathrm{M}$ dwarfs, and that $\mathrm{M}$ dwarfs appear to have a lower planet occurrence rate overall. 


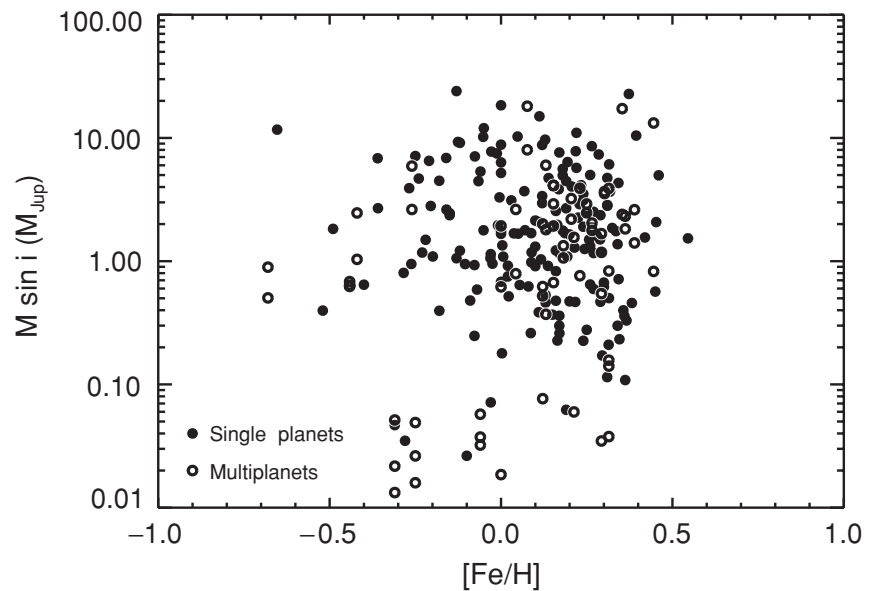

Figure 28. Plot of $[\mathrm{Fe} / \mathrm{H}]$ vs. $M \sin i$. Filled circles are single-planet systems, and open circles represent multiplanet systems.

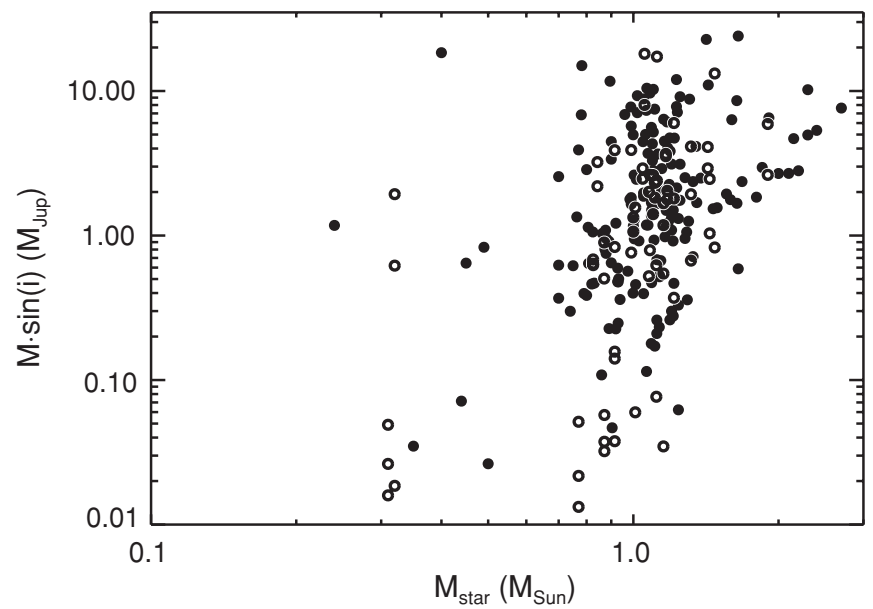

Figure 29. $M \sin i$ vs. stellar mass. Filled circles are single-planet systems, and open circles represent multiplanet systems. Apparently, minimum planet mass does not correlate well with stellar mass in the range of 0.3-1.9 $M_{\odot}$.

\subsection{Semimajor Axis versus Stellar Mass}

We plot semimajor axis versus stellar mass (Figure 30). Two features are readily apparent in this plot: a lack of closein planets orbiting stars with $M>1.5 M_{\odot}$, and a lack of long-period planets orbiting stars with $M<0.5 M_{\odot}$. Thus, the semimajor axes of giant planets correlate positively and sensitively with stellar mass. The first of these features has already been noted by Johnson et al. (2007a), who find that the effect is statistically significant. The lack of long-period planets around $\mathrm{M}$ dwarfs is puzzling, since significant numbers of $\mathrm{M}$ dwarfs have been a part of the major radial velocity planet searches since at least 1995, sufficient to detect any massive long-period planets at orbital distances of a few AU. We are undertaking a more thorough study of the occurrence rate of long-period planets around all of our targets to confirm the reality of the apparent dearth of long-period planets around lowmass stars. Nonetheless, a correlation seems to be emerging, driven primarily by the lack of long-period planets orbiting M dwarfs.

\subsection{Mass Ratio}

For the multiplanet systems, we plotted the ratio of the minimum mass of the outermost planet to the minimum mass

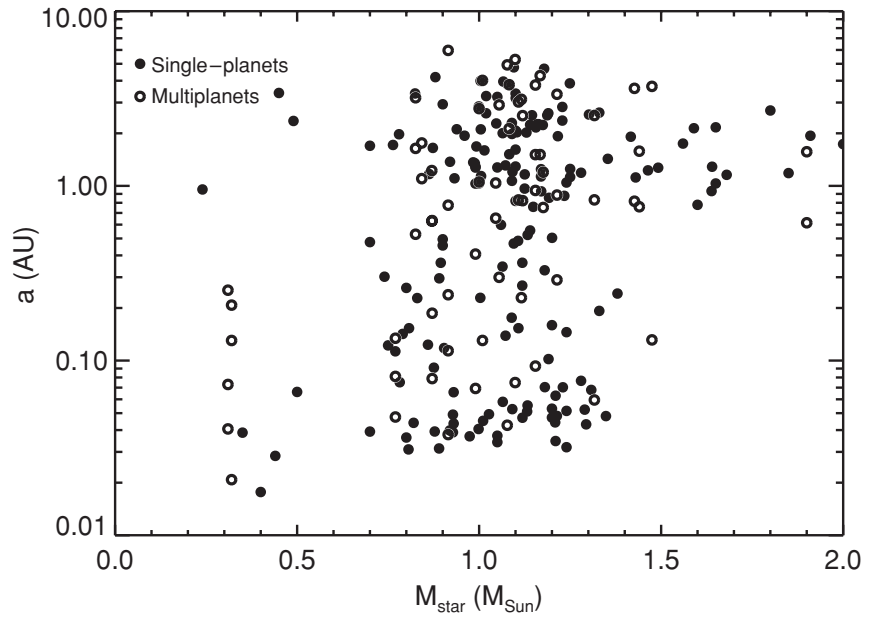

Figure 30. Plot of $a$ vs. stellar mass. Filled circles are single-planet systems, and open circles are multiplanet systems. There is no strong correlation among planets orbiting stars with $0.8<M_{\odot}<1.3$, but planets around the lowest-mass stars have smaller semimajor axes.

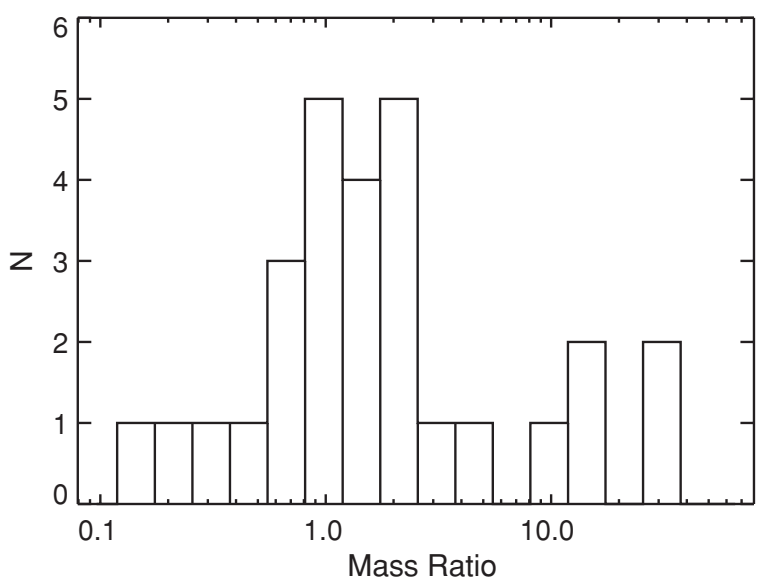

Figure 31. Distribution of $M \sin i$ ratios computed as $M \sin i$ (outer) $/ M \sin i$ (inner) for the outermost two planets in the 20 known multiplanet systems.

of the next outermost planet $(M \sin i$ (outer) $/ M \sin i$ (inner)) as shown in Figure 31. The distribution peaks near 1 and appears somewhat skewed toward systems in which the outer planet is more massive. However, the $a^{-\frac{1}{2}}$ dependence of orbital distance on reflex amplitude undoubtedly plays an important role, since low-mass planets are more easily detected closer to their parent star. While it is true that detected outer planets tend to be more massive, this may not reflect the actual distribution of planet masses in multiple-planet systems.

\section{SUMMARY AND DISCUSSION}

A major outstanding question in planet formation remains: how common are planetary systems with architectures similar to the solar system? In the known planetary systems within 200 pc, the median $M \sin i$ is $1.6 M_{\mathrm{Jup}}$, and the median orbital distance is 0.9 AU. Given the observational biases, these numbers suggest that the solar system may yet prove to be typical.

The primary way in which the solar system appears unusual is in the nearly circular orbits of its planets, since the median eccentricity of the detected exoplanets is 0.25 (excluding tidally circularized planets). Figure 18, however, shows that these high eccentricities are generally restricted to planets with $M \sin i>1 M_{\text {Jup }}$, and that nearly circular or- 
bits are typical in planetary systems having no planets with $M>1 M_{\text {Jup }}$.

We have also shown that there are four classes of planetary systems with distinct orbital distance distributions - (1) planetary systems around stars with $M>1.5 M_{\odot},(2)$ those with $M<0.5 M_{\odot}$, (3) other apparently single systems, and (4) multiple-planet systems. ${ }^{13}$ This provides a challenge for planet formation and migration theories to reproduce these disparate distributions.

The 1 AU jump in the orbital distance distribution (Figure 9) of planets orbiting single stars may correspond to the planet formation ice line, or at least constitute an observational constraint on its location. It also suggests the existence of a large, yet unobserved population of giant planets beyond $1 \mathrm{AU}$. The next decade of radial velocity planet detection will reveal the population of giant plants from 3 to $7 \mathrm{AU}$, and thus provide a census of giant planets which have not experienced significant inward migration. This provides theory with an opportunity not only to explain the above observations, but also to provide observational tests of those explanations by predicting the frequency of such in situ planets orbiting various classes of stars.

The authors acknowledge and thank R. Paul Butler and Steven S. Vogt for their major contributions in obtaining the radial velocity measurements herein, without which this work would not have been possible. The authors also wish to acknowledge helpful discussions with many astronomers for providing and refining many of the ideas of this paper. An incomplete list would include Alan Boss, Eric Agol, Kristen Menou, James Kasting, Rory Barnes, and Ed Thommes. The work herein is based on observations obtained at the W. M. Keck Observatory, which is operated jointly by the University of California and the California Institute of Technology. The Keck Observatory was made possible by the generous financial support of the W.M. Keck Foundation. We wish to recognize and acknowledge the very significant cultural role and reverence that the summit of Mauna Kea has always had within the indigenous Hawaiian community. We are most fortunate to have the opportunity to conduct observations from this mountain. This research has made use of the SIMBAD database, operated at CDS, Strasbourg, France, and of NASA's Astrophysics Data System Bibliographic Services, and is made possible by the generous support of NASA and the NSF, including grant AST-0307493. J.T.W received support from NSF grant AST-0504874. G.W.M. received support from NASA grant NNG06AH52G, and D.A.F from NASA grant NNG05G164G and the Cottrell Science Scholar Program. E.B.F acknowledges the support of NASA RSA 1326409. J.A.J is supported by NSF grant AST-0702821. The authors acknowledge the University of Florida HighPerformance Computing Center for providing computational resources and support that have contributed to the results reported in this paper.

\section{APPENDIX}

\section{STATISTICAL METHODS: K-S TESTS}

In this work, we used the $\mathrm{K}-\mathrm{S}$ statistical test, based on the two-sided $D$-statistic, to measure the likelihood that any apparent differences in distributions (for a given property) were in fact attributable to chance (Knuth 1997). Considering

\footnotetext{
13 There is, at present, only slight overlap between these classes: GJ 581 and
} GJ 876 are both multiple-planet systems and have $M<0.5 M_{\odot}$. any independent variable, " $x$," we construct two CDFs of that variable, one for planets in single-planet systems, $F_{s}(x)$, and another for planets in multiplanet systems, $F_{m}(x)$. We define the $D$-statistic as follows:

$$
\begin{aligned}
& D^{+}=\max \left(F_{m}(x)-F_{s}(x)\right), \\
& D^{-}=\max \left(F_{s}(x)-F_{m}(x)\right) .
\end{aligned}
$$

This statistic measures the maximum difference between the two CDF graphs being compared, with $D^{+}$measuring how high the multiplanet $\mathrm{CDF}$ rises above the single-planet CDF, and $D^{-}$measuring how high the single-planet CDF rises above the multiplanet CDF. A large $D^{+}$implies that multiplanet systems systematically have lower values of $x$, and a large $D^{-}$implies that they have higher values. We quantify this using a Monte Carlo method to produce a $\mathrm{K}-\mathrm{S}$ confidence value for this statistic.

To establish confidence levels, we count $n$ planets in singleplanet systems and $m$ planets in multiplanet systems. The " $D$ " statistic measures how frequently $m$ planets drawn with replacement from the distribution of $x$ for single-planet systems will have $D_{\text {simulated }}^{+}$and $D_{\text {simulated }}^{-}$as large or larger than the $D$-statistics for the actual multiplanet systems. We simulated 100,000 multiplanet distributions using the distribution for single-planet systems, and the fraction of trials with $D_{\text {simulated }}^{+}$ greater than the $D^{+}$for multiplanet systems is the $\mathrm{K}-\mathrm{S}$ test $p$ value $(p)$ that the CDF for multiplanet systems lies above the $\mathrm{CDF}$ for single-planet systems - and similarly, the fraction of trials with $D_{\text {simulated }}^{-}$greater than the $D^{-}$for multiplanet systems is the $\mathrm{K}-\mathrm{S}$ test $p$-value that the $\mathrm{CDF}$ for multiplanet systems lies below the CDF for single-planet systems. The $\mathrm{K}-\mathrm{S}$ test does not assume any particular shape for the distributions, however, we have assumed that the counts follow Poisson statistics.

\section{REFERENCES}

Aarseth, S. J., Lin, D. N. C., \& Palmer, P. L. 1993, ApJ, 403, 351

Barnes, R., \& Quinn, T. 2004, ApJ, 611, 494

Barnes, R., Goździewski, K., \& Raymond, S. N. 2008, ApJ, 680, L57

Bean, J. L., McArthur, B. E., Benedict, G. F., \& Armstrong, A. 2008, ApJ, 672, 1202

Bodenheimer, P., Laughlin, G., \& Lin, D. N. C. 2003, ApJ, 592, 555

Bouchy, F., et al. 2008, A\&A, in press

Bryden, G., Różyczka, M., Lin, D. N. C., \& Bodenheimer, P. 2000, ApJ, 540 1091

Butler, R. P., \& Marcy, G. W. 1996, ApJ, 464, L153

Butler, R. P., Marcy, G. W., Fischer, D. A., Brown, T. M., Contos, A. R., Korzennik, S. G., Nisenson, P., \& Noyes, R. W. 1999, ApJ, 526, 916

Butler, R. P., Marcy, G. W., Vogt, S. S., \& Apps, K. 1998, PASP, 110, 1389

Butler, R. P., et al. 2006, ApJ, 646, 505

Chambers, J. E. 1999, MNRAS, 304, 793

Chiang, E. I., \& Murray, N. 2002, ApJ, 576, 473

Cochran, W. D., Endl, M., Wittenmyer, R. A., \& Bean, J. L. 2007, ApJ, 665, 1407

Correia, A. C. M., Udry, S., Mayor, M., Laskar, J., Naef, D., Pepe, F., Queloz, D., \& Santos, N. C. 2005, A\&A, 440, 751

Cresswell, P., \& Nelson, R. 2006, A\&A, 450, 833

Cumming, A., Butler, R. P., Marcy, G. W., Vogt, S. S., Wright, J. T., \& Fischer, D. A. 2008 , PASP, 120,531

D’Angelo, G., Kley, W., \& Henning, T. 2003, ApJ, 586, 540

Desort, M., Lagrange, A., Galland, F., Beust, H., Udry, S., Mayor, M., \& Lo Curto, G. 2008, AAP, 491, 883

Eggenberger, A., Udry, S., Chauvin, G., Beuzit, J.-L., Lagrange, A.-M., Ségransan, D., \& Mayor, M. 2007, A\&A, 474, 273

Endl, M., Cochran, W. D., Kürster, M., Paulson, D., Wittenmyer, R. A., MacQueen, P. J., \& Tull, R. G. 2006, ApJ, 649, 436

Fischer, D. A., Marcy, G. W., Butler, R. P., Laughlin, G., \& Vogt, S. S. 2002a, ApJ, 564, 1028

Fischer, D. A., Marcy, G. W., Butler, R. P., Vogt, S. S., \& Apps, K. 1999, PASP, 111,50 
Fischer, D. A., Marcy, G. W., Butler, R. P., Vogt, S. S., Frink, S., \& Apps, K. 2001, ApJ, 551, 1107

Fischer, D. A., Marcy, G. W., Butler, R. P., Vogt, S. S., Walp, B., \& Apps, K. 2002b, PASP, 114, 529

Fischer, D. A., \& Valenti, J. 2005, ApJ, 622, 1102

Fischer, D. A., et al. 2008, ApJ, 675, 790

Ford, E. B. 2006, in ASP Conf. Ser. 352, New Horizons in Astronomy: Frank N. Bash Symp., ed. S. J. Kannappan, S. Redfield, J. E. Kessler-Silacci, M. Landriau, \& N. Drory (San Francisco, CA: ASP), 15

Ford, E. B., Lystad, V., \& Rasio, F. A. 2005, Nature, 434, 873

Ford, E. B., \& Rasio, F. A. 2008, ApJ, 686, 621

Ford, E. B., Rasio, F. A., \& Sills, A. 1999, ApJ, 514, 411

Gaudi, B. S., et al. 2008, Science, 319, 927

Goldreich, P., \& Sari, R. 2003, ApJ, 585, 1024

Gregory, P. C. 2007, MNRAS, 381, 1607

Ida, S., \& Lin, D. N. C. 2004, ApJ, 616, 567

Ida, S., \& Lin, D. N. C. 2008, ApJ, 685, 584

Johnson, J. A., Butler, R. P., Marcy, G. W., Fischer, D. A., Vogt, S. S., Wright, J. T., \& Peek, K. M. G. 2007a, ApJ, 670, 833

Johnson, J. A., Marcy, G. W., Wright, J. T., Driscoll, P., Butler, R. P., Hekker, S., Reffert, S., \& Vogt, S. S. 2007b, ApJ, 665, 785

Kley, W., Peitz, J., \& Bryden, G. 2004, A\&A, 414, 735

Kley, W., Lee, M. H., Murray, N., \& Peale, S. J. 2005, A\&A, 437, 727

Knuth, D. E. 1997, The Art of Computer Programming, Vol. 2 (Reading, MA: Addison-Wesley)

Kokubo, E., \& Ida, S. 2002, ApJ, 581, 666

Konacki, M., Torres, G., Sasselov, D. D., \& Jha, S. 2003, ApJ, 597, 1076

Laughlin, G., \& Chambers, J. E. 2002, AJ, 124, 592

Lee, M. H., Butler, R. P., Fischer, D. A., Marcy, G. W., \& Vogt, S. S. 2006, ApJ, 641,1178

Lee, M. H., \& Peale, S. J. 2003, ApJ, 592, 1201

Levison, H. F., Lissauer, J. J., \& Duncan, M. J. 1998, AJ, 116, 1998

Lovis, C., et al. 2006, Nature, 441, 305

Marcy, G. W., Butler, R. P., Fischer, D., Vogt, S. S., Lissauer, J. J., \& Rivera, E. J. 2001, ApJ, 556, 296

Marcy, G. W., Butler, R. P., Vogt, S. S., Fischer, D. A., Henry, G. W., Laughlin, G., Wright, J. T., \& Johnson, J. A. 2005, ApJ, 619, 570

Marzari, F., Scholl, H., \& Tricarico, P. 2005, in 36th Ann. Lunar and Planetary Sci. Conf., ed. S. Mackwell \& E. Stansbery, 1289

Mayor, M., \& Queloz, D. 1995, Nature, 378, 355
Mayor, M., Udry, S., Naef, D., Pepe, F., Queloz, D., Santos, N. C., \& Burnet, M. 2004, A\&A, 415, 391

Mayor, M., et al. 2009, AAP, 493, 639

McArthur, B. E., et al. 2004, ApJ, 614, L81

Murray, N. 2003, in ASP Conf. Ser. 294, Scientific Frontiers in Research on Extrasolar Planets, 165

Naef, D., Mayor, M., Beuzit, J. L., Perrier, C., Queloz, D., Sivan, J. P., \& Udry, S. 2004, A\&A, 414, 351

Nelson, R. P., \& Papaloizou, J. C. B. 2002, MNRAS, 333, L26

Niedzielski, A., Gozdziewski, K., Wolszczan, A., Konacki, M., Nowak, G., \& Zieliński, P. 2008, arXiv:0810.1710

Papaloizou, J. C. B. 2005, Celestial Mech. Dyn. Astron., 91, 33

Pepe, F., Bouchy, F., Queloz, D., \& Mayor, M. 2003, in ASP Conf. Ser. 294 Scientific Frontiers in Research on Extrasolar Planets, ed. D. Deming \& S Seager (San Francisco, CA: ASP), 39

Pepe, F., et al. 2007, A\&A, 462, 769

Pollack, J. B., Hubickyj, O., Bodenheimer, P., Lissauer, J. J., Podolak, M., \& Greenzweig, Y. 1996, Icarus, 124, 62

Raghavan, D., Henry, T. J., Mason, B. D., Subasavage, J. P., Jao, W.-C., Beaulieu, T. D., \& Hambly, N. C. 2006, ApJ, 646, 523

Rasio, F. A., Tout, C. A., Lubow, S. H., \& Livio, M. 1996, ApJ, 470, 1187

Rivera, E. J., et al. 2005, ApJ, 634, 625

Sándor, Z., \& Kley, W. 2006, A\&A, 451, L31

Santos, N. C., Israelian, G., \& Mayor, M. 2001, A\&A, 373, 1019

Santos, N. C., et al. 2004, A\&A, 426, L19

Takeda, G., Ford, E. B., Sills, A., Rasio, F. A., Fischer, D. A., \& Valenti, J. A. 2007, ApJS, 168, 297

Tanaka, H., \& Ward, W. R. 2004, ApJ, 602, 388

Tinney, C. G., Butler, R. P., Marcy, G. W., Jones, H. R. A., Laughlin, G., Carter, B., Bailey, J. A., \& O'Toole, S. 2006, ApJ, 647, 594

Trilling, D. E., Lunine, J. I., \& Benz, W. 2002, A\&A, 394, 241

Udry, S., et al. 2007, A\&A, 469, L43

Valenti, J. A., \& Fischer, D. A. 2005, ApJS, 159, 141

Vogt, S. S., Butler, R. P., Marcy, G. W., Fischer, D. A., Henry, G. W., Laughlin, G., Wright, J. T., \& Johnson, J. A. 2005, ApJ, 632, 638

Wisdom, J., \& Holman, M. 1991, AJ, 102, 1528

Wittenmyer, R. A., Endl, M., \& Cochran, W. D. 2007, ApJ, 654, 625

Wolszczan, A., \& Frail, D. A. 1992, Nature, 355, 145

Wright, J. T. 2005, PASP, 117, 657

Wright, J. T., \& Howard, A. W. 2008, ApJS, submitted

Wright, J. T., et al. 2007, ApJ, 657, 533 